«Was it a vision, or a waking dream? » Constellations intérieures, incandescences du sensible : Bright Star de Jane Campion ou la nuit étoilée de Keats

\title{
Esra Aykin
}

\section{OpenEdition}

\section{Journals}

Édition électronique

URL : https://journals.openedition.org/recherchestravaux/673

DOI : 10.4000/recherchestravaux.673

ISSN : 1969-6434

Éditeur

UGA Éditions/Université Grenoble Alpes

Édition imprimée

Date de publication : 1 novembre 2014

Pagination : 15-53

ISBN : 978-2-84310-282-0

ISSN : 0151-1874

Référence électronique

Esra Aykin, « «Was it a vision, or a waking dream? » Constellations intérieures, incandescences du sensible : Bright Star de Jane Campion ou la nuit étoilée de Keats », Recherches \& Travaux [En ligne], 84 2014, mis en ligne le 01 avril 2016, consulté le 29 octobre 2021. URL : http://journals.openedition.org/ recherchestravaux/673; DOI : https://doi.org/10.4000/recherchestravaux.673 


\section{"Was it a vision, or a waking dream?" Constellations intérieures, incandescences du sensible : Bright Star de Jane Campion ou la nuit étoilée de Keats}

Je me mis à chercher dans le ciel une étoile, que je croyais connaître, comme si elle avait quelque influence sur ma destinée. L'ayant trouvée, je continuai ma marche en suivant les rues dans la direction desquelles elle était visible, marchant pour ainsi dire au-devant de mon destin, et voulant apercevoir l'étoile jusqu'au moment où la mort devait me frapper.

Gérard DE NervaL ${ }^{\mathrm{I}}$

En s'immergeant des années durant dans l'univers du poète romantique anglais John Keats (I795-I82I), Jane Campion prenait la mesure, dans le même temps, de la poignante richesse d'une vie et d'une ouvre vouées à la poésie, d'une impérieuse nécessité de porter celles-ci à l'écran, et de l'indéniable difficulté de la tâche. C'est en effet à la faveur de l'un de ces hasards - ou de ces heureuses synchronicités - dont la vie est poétiquement parcourue que la réalisatrice néo-zélandaise, qui ne cache pas ses préventions initiales contre la poésie, est soudainement confrontée à celui dont elle dira plus tard : "Les poèmes de Keats furent mes portes d'entrée dans la poésie, et sa vie et ses lettres, une invitation à faire l'expérience d'une relation créative à moi-même ravivée,

I. G. de Nerval, Les Filles du feu suivi de Aurélia, Paris, Gallimard, coll. «Folio classique», I997, p. 295. 
ainsi qu'à avoir foi dans le divin ; il n'y a pas d'autre explication à sa meilleure poésie ${ }^{2}$ ", évoquant "cette voie d'accès à $[s]$ es sens, $[s]$ on âme et $[s]$ on imagination ouverte par Keats, une porte donnant sur le cœur humain ${ }^{3}$.» À l'origine de cet éblouissement fondateur se trouve l'impressionnante biographie consacrée à Keats par Andrew Motion ${ }^{4}$, dont la lecture, presque fortuite, marque un tournant pour la cinéaste, faisant germer dans son esprit le vif désir d'un film. C'est tout particulièrement le dernier tiers de cette biographie qui agit comme un révélateur, dans lequel se trouve dépeinte la passion lumineuse et tragique qui unira deux ans et demi durant Keats et sa voisine Fanny Brawne : «je suis tombée amoureuse de leur histoire d'amour, bien plus passionnée, puissante que celle de Roméo et Juliette. Elle est tout en vérité, en justesse, en innocence, en souffrance. Un pur amour dont ils paieront le prix ${ }^{5} »$. Dès lors, la cinéaste n'aura de cesse de se pencher sur l'œuvre et la vie de Keats, s'imprégnant tour à tour de sa poésie et de sa correspondance, dont la magnifique trentaine de lettres d'amour à Fanny. Si l'évidence des affinités fécondes est bien là, esquissant déjà une possible passerelle entre poésie et cinéma, le projet de film ne restera longtemps qu'un rêve :

Je me prenais à me demander si je pouvais raconter en quelque manière son histoire dans un film, pour me ressaisir aussitôt. Plus personne ne lit vraiment de poésie, mais le coup le plus terrible porté à mon espérance était tout simplement le fait que lorsque je lisais les poèmes je ne les comprenais pas complètement, ou, dans le cas des longs poèmes «Endymion» ou «Hyperion », ignorais les références classiques. Comment pouvais-je faire un film sur Keats si je ne comprenais pas la poésie ${ }^{6}$ ?

2. "Keats's poems were my portals into poetry and his life and letters staged for me a revived creative relationship with myself as well as faith in the divine; there is no other explanation for his best poetry." (J. Campion, "My John Keats", introduction, dans J. Keats, "Bright Star": The Complete Poems and Selected Letters, Londres, Vintage Books, coll. "Vintage Classics», 2009, p. XVI; je traduis.)

3. "[...] this pathway Keats has opened into my senses, my soul and my imagination, a portal to the human heart» (ibid.; je traduis).

4. A. Motion, Keats, Londres, Faber and Faber, 1998. A. Motion sera par ailleurs conseiller littéraire sur Bright Star.

5. J. Campion, "Une histoire d'amour plus forte que Roméo et Juliette» [en ligne], entretien avec E. Frois, Le Figaro. Disponible sur <http://www.lefigaro.fr/festival-decannes/2009/05/15/030II-200905I5ARTFIGooo03-une-histoire-d-amour-plus-forte-que-romeoet-juliette-.php> (consulté le 26/06/20I4).

6. "I drifted into wondering if I could somehow tell his story on film, only to shake myself. Nobody really reads poetry anymore, but the cruellest blow to my hope was simply that while I was reading the poems I didn't completely understand them, or, in the case of the long poems Endymion or Hyperion, I didn't know the classical references. How could I make a film about Keats if I didn't understand poetry?» (J. Campion, "My John Keats», dans "Bright Star": The Complete Poems and Selected Letters, ouvr. cité, p. XIII-XIV; je traduis.) 
Cependant, l'idée suivait son cours souterrain. Deux ans plus tard, au bord de l'eau, Campion songe à Keats et Fanny, dans la complicité d'une brise annonciatrice de celles qui traverseront le film à venir : «La chaleur du soleil était comme un baiser. La vie se ralentissait : une brise [...] arriva comme un événement ${ }^{7}$." Elle se replonge dans la lecture des poèmes de Keats - dont l'une des fameuses odes, l'«Ode sur l'indolence», caractérisée précisément par cet «état rêveur, flottant ${ }^{8}$ » qui est alors celui de la cinéaste - pour réaliser que, si le sens parfois lui échappe, l'envoûtement de cette poésie n'en est pas moins opérant :

La séduction par les mots, le rythme, l'atmosphère et l'intimité avait commencé. J'aimais que ces mots, ces sons et ces glissements de sens puissent s'entrelacer comme des guirlandes de pâquerettes, comme les courants des rivières, comme des murmures capables, sous les doigts de Keats, de me décrire à moi-même tout en ayant une présence sensuelle et délicieuse qui s'imposait à mes sensations? .

\section{Romantiser le monde}

Cette perception non mentale, cette immersion au cœur même de la vérité poétique par l'intermédiaire de la sensation et de l'intuition, agissant comme une illumination, signe sans aucun doute la véritable naissance de Bright Star (sorti en 2009), ou plutôt l'heure secrète de sa conception. Elle est comme une réponse intime à ce qui constitue la quintessence de toute poésie et dont, à son tour, en écho, le film sera entretissé - ce que Keats appelait «negative capability", cette "capacité négative» qui est pur accueil du monde en soi, dans toute son heureuse inexplicabilité, son insondabilité, et qu'il définira dans sa lettre du 2I décembre I8I7 à ses frères George et Tom :

J'ai été frappé tout d'un coup de la qualité essentielle à la formation d'un Homme d'Art accompli particulièrement en Littérature et que Shakespeare possédait à un degré si énorme - je veux dire la Capacité Négative, je veux dire celle de demeurer au sein des incertitudes, des Mystères, des doutes, sans s'acharner à chercher le fait et la raison [...] : chez un grand poète, le sens de la Beauté l'emporte sur toute autre considération, ou plutôt oblitère toute considération ${ }^{10}$.

7. "The sun's warmth felt like a kiss. Life slowed down: a breeze [...] arrived as an event." (Ibid., p. xIV; je traduis.)

8. "[...] the dreamy drifty state [I was enjoying]" (ibid.).

9. "[...] the seduction by words, rhythm, atmosphere and intimacy had begun. I was loving that these words, sounds and drifts of meaning could be joined like daisy chains, like streams to rivers, like whispers that could in Keats's hands, describe me to myself and all the while have a sensuous and delicious presence that played on my sensations" (ibid.; je traduis).

Io. J. Keats, Lettres à Fanny et autres correspondances, trad. R. Davreu, Paris, Belin, 20Io, p. 96. 
Cette perméabilité au mystère d'être, cet hymne vibrant à la Beauté représentent le cœur absolu de la poésie keatsienne, et une clé pour entrer dans Bright Star, tant il est vrai qu'ils imprègnent tout le film, en une subtile osmose. La nouvelle perspective qui s'ouvre pour Jane Campion au détour de cette lettre sera en ce sens décisive : "Je commençai à réaliser que la poésie n’exigeait de nous peut-être pas tant de la comprendre que de l'aimer, ou d'être charmé, séduit, intrigué ou impressionné par elle ${ }^{\mathrm{II}}$.» Cette prise de conscience amorcera la rédaction au long cours d'un scénario dans lequel il ne pouvait s'agir d'adopter le point de vue habituel d'une biographie filmée : "vouloir illustrer au cinéma un tel vertige de sentiments est délicat. [...] Je me suis mise au travail pendant dix-huit mois avec une seule idée en tête : raconter l'histoire d'un amour incandescent, fulgurant comme le poème de Keats, "Bright Star", étoile brillante et lointaine ${ }^{\mathrm{I2}}$ ", déclare la réalisatrice.

Faire exister à l'écran la présence d'un poète et de sa poésie aurait pu passer par une représentation classique, centrée sur la narration plus ou moins exhaustive et fidèle des données biographiques, par une mise en scène où prédomineraient l'extériorité, la succession des faits et gestes d'un personnage - et de son entourage - dont l'identité poétique ne serait perceptible qu'au travers çà et là d'un geste d'écriture, d'un poème déclamé, où la poésie n'interviendrait en somme qu'à titre d'illustration, se juxtaposant à la trame événementielle. Le film aurait pu également, suivant des sentiers moins empruntés, sur lesquels se sont aventurés en éclaireurs par exemple Tarkovski, Angelopoulos, Ruiz ou les frères Taviani, traduire cinématographiquement l'identité plus profonde, plus secrète d'un poète en manifestant son regard intérieur, extériorisation filmique des images qui peuplent l'imaginaire poétique ${ }^{\mathrm{r}}$. Jane Campion laissera d'ailleurs parfois émerger de telles images intérieures. Mais, si c'est aussi sur le regard qu'elle axe sa quête, c'est un autre chemin qu'elle choisit d'emprunter, afin de rendre - et de rendre grâce à - la poésie keatsienne, verbale, et dans le même temps celle, intrinsèque, de ces sentiments, de ces êtres, de cette situation. D’une part, elle décide de regarder toute l'histoire - et donc Keats lui-même ainsi que sa poésie - par les yeux de Fanny; et d'autre part, elle invente sa propre

II. "I began to realise that perhaps poetry is not so much in need of understanding as loving, or being enchanted, seduced, intrigued or awed." (J. Campion, "My John Keats», dans "Bright Star": The Complete Poems and Selected Letters, ouvr. cité, p. xiv; je traduis.)

I2. J. Campion, «Un amour incandescent d'un romantisme absolu» [en ligne], entretien du 06/or/20Io, Le Figaro. Disponible sur <http://www.lefigaro.fr/cinema/2010/or/o6/0300220I00I06ARTFIG00455-jane-campion-un-amour-incandescentd-un-romantisme-absolu-.php> (consulté le 25/06/20I4).

I3. Voir à ce sujet mon étude " "Ses yeux toujours tournés vers le dedans." Visages et paysages intérieurs de l'écrivain au cinéma", dans Cinéma et Littérature. Le Grand Jeu 2, J.-L. Leutrat (dir.), Grenoble, De l'Incidence éditeur, 20II, p. 319-363. 
poétique filmique, alliée à la poésie de Keats, en osmose avec celle-ci, dans un accord intime qui se situe bien au-delà de la seule citation, de la seule voix off, du seul geste de la plume courant sur le papier, pour imprégner l'essence même du film de toute l'âme de Keats, avec celle de Fanny en filigrane - jusqu'à devenir tout entier ce "véhicule de poésie» que Jean Cocteau voyait dans le cinéma. Les acteurs auxquels elle fait appel pour incarner les deux jeunes gens - Abbie Cornish-Fanny et Ben Wishaw-Keats - sont une merveilleuse caisse de résonance, aux accents infiniment subtils, à cette poétique du regard.

C'est à la confluence du regard sur le monde et du regard intérieur que naît la beauté. Keats, tout au long de sa quête éperdue de celle-ci - «j'ai aimé le principe de la Beauté en toutes choses ${ }^{14}$ ", résumera-t-il dans une lettre à Fanny de février 1820 -, n'a eu de cesse de le rappeler. Ainsi, dans une lettre de mars I8I8 à Benjamin Bailey, il évoque ces "Choses semi-réelles telles que l'Amour, les Nuages, etc., qui requièrent un accueil de l'Esprit pour les faire exister pleinement - et Nonchoses qui acquièrent Grandeur et dignité par une quête $\operatorname{ardente}^{15}$ ». Comme le remarque Andrew Motion, ces déclarations et d'autres allant dans le même sens, "sur la fusion de l'objet et de l'esprit, et sur l'éveil d'une réalité extérieure en une "Véritê" incarnée en "Beauté" ", tendent à rappeler que "cette "Beauté" [...] n'est pas la cohérence de la chose en soi, mais une union de l'observable et du monde intérieur, une synthèse qui dépend des effets combinés des sens, du "gusto", et du théâtre de l'imagination ${ }^{16}$ ». "La beauté est vérité, la vérité beautét ${ }^{17}$ ", énonçait un vers de l'"Ode sur une urne grecque» ("Ode on a Grecian Urn»). Ce plein accueil de la beauté-vérité, ce processus créatif qui requiert une participation de l'être en sa totalité - la capacité négative chère à Keats -, Jane Campion en fait la source vive de Bright Star, le cœur irradiant de l'étoile. À son tour, son regard de cinéaste opère cette synthèse de l'univers sensible - d'un visible réinventé par la vision même -, et du monde intérieur keatsien. Il est regard poétique en ce sens qu'il ne se contente pas d'enregistrer une poésie extérieure à lui, d'en prendre acte, mais participe de cette romantisation $d u$ monde qu'est la poésie, processus transfigurateur appelé de ses vœux par Novalis : «Le monde doit être romantisé. [...] Lorsque je donne à l'ordinaire un sens élevé, au commun

I4. J. Keats, Lettres à Fanny et autres correspondances, ouvr. cité, p. 550.

I5. Ibid., p. I39.

16. "[...] about the fusion of object and mind, and about the awakening of external reality into a "Truth" which is embodied as "Beauty". This "Beauty" [...] is not the coherence of a thing in itself, but a union of the observable and the interior world, a synthesis which depends on the interplay of senses, on "gusto", and on imaginative drama" (A. Motion, Keats, ouvr. cité, p. 218; je traduis).

I7. " "Beauty is truth, truth beauty" [...]» (J. Keats, "Bright Star": The Complete Poems and Selected Letters, ouvr. cité, p. 240 ; je traduis). 
un aspect mystérieux, au connu la dignité de l'inconnu, au fini l'apparence de l'infini, alors je les romantise ${ }^{18}$.» Romantisation du monde ou capacité négative, l'acte poétique, ou ciné-poétique, est toujours acte alchimique, geste intérieur permettant de faire accéder la réalité immédiate - en une "potentialisation qualitative ${ }^{19}$ " discernée par Novalis, comme en écho à la beauté-vérité célébrée par Keats -, à sa véritable essence, de la transfigurer en ce Réel qui attendait d'être ainsi révélé, et que le poète romantique allemand appréhende en ces termes : "La poésie est le véritable réel absolu. [...] Plus c'est poétique, plus c'est vrai ${ }^{20}$."

\section{"C'est une ondée inépuisable / De lumière que la poésie ${ }^{2 \mathrm{II}}$ "}

L'exaltation tout en nuances de la lumière au long du film, de clairs-obscurs en intenses solarités, de trop-pleins lyriques en lueurs secrètes, représente l'un des éléments clés de cette poétique filmique accordée à celle de Keats et à l'absolu des sentiments, traduisant, dans ses modulations intimistes, le langage même de l'âme amoureuse. La cinéaste précise en ce sens :

À mes yeux, le monde de Keats et de Fanny est empreint de lumière; il s'illumine littéralement et irradie, et même si Keats disparait à la fin, la flamme allumée par son génie de la poésie et son esprit unique ne peut être éteinte. Telle est l'ambition de Bright Star: sensibiliser le public à cette lumière, allumer la flamme ${ }^{22}$.

Cette vibration à la fois puissante et discrète de la lumière, en ses infinies variations, s'accompagne d'une attention toute particulière à ses réceptacles privilégiés : bien évidemment la nature, dont les changements, entre effloraisons radieuses et désolations hivernales, épousent à merveille les saisons de l'âme - accomplissant en cela une aspiration essentielle du romantisme -; mais aussi certains éléments intérieurs auxquels leur valeur même de passage entre dedans et dehors, visible et invisible, confère une forte dimension poétique - ce sont toutes les fenêtres, rideaux, miroirs dans lesquels viennent se prendre et se refléter la vie, puis la mort. La lumière joue aussi sur les visages, y laissant transparaître les notes les plus secrètes de l'être, entre joie et déchirure. Gravité

18. Novalis, Le monde doit être romantisé, Paris, Allia, 2002, p. 46.

19. Ibid.

20. "Die Poesie ist das echt absolut Reelle. [...] Je poetischer, je wahrer.» (Novalis, Fragments/ Fragmente, Paris, Aubier Montaigne, coll. «Bilingue», 1973, p. II6-II7; je modifie la traduction.)

2I. J. Keats, "Sommeil et Poésie», dans Poèmes et poésies, trad. P. Gallimard, Paris, Gallimard, coll. «Poésie», 2013, p. 42-43.

22. J. Campion, dans dossier de presse cité, p. 4. Signalons le remarquable travail du directeur de la photo G. Fraser, caractérisé pour la cinéaste par «une sorte de tendresse dans la mise en lumière» (ibid., p. Io). 
grandissante du regard de Fanny, hanté, de l'autre côté d'une belle saison éphémère, de douleurs inexprimables. Mais aussi, en miroir, ferveur inquiète de celui du poète livré lui aussi à tous les insondables, et le jeu infiniment nuancé des regards amoureux entre eux, qui en disent long là où les mots n'ont pas le droit d'éclore, ou - sauf dans la langue du poème - ne peuvent se tenir qu'en deçà du vertige. Le regard, enfin, porté par l'entourage proche sur cet amour naissant, brûlant et survivant à la mort même. Incroyables regards de la petite Margaret, dite Toots, et de Samuel, dit Sam, la sœur et le frère cadets de Fanny qui, convenances obligent, l'accompagnent toujours, à quelques pas de distance, de leur présence ténue mais dense : ils sont déjà là lorsque Fanny lit pour la première fois la poésie de Keats, dans le recueil Endymion fraîchement rapporté de la librairie à sa demande. Ils sont toujours là à l'heure du désastre, de l'annonce de l'irréparable, la mort redoutée, au loin, du bienaimé - Sam deviné à l'arrière-plan, Toots dans la douloureuse sidération d'un plan rapproché révélant son regard perdu dans le vide, empli de la terreur de quelque chose de trop grand pour elle, tandis que, hors champ, les sanglots, les appels lancinants de Fanny déchirent la nuit d'hiver. Regards posés sur elle, ensemble ou tour à tour, consignant dans leurs profondeurs gestes, mots, silences, perceptions et événements dans le sillage muet d'un amour dont ils marquent les étapes majeures, d'une main tenue pour la première fois à une bague de fiançailles discrètement glissée au doigt de l'aimée, d'une longue attente angoissée à la lettre reçue dans l'euphorie amoureuse ou le désespoir, d'une terrible visite au jeune frère agonisant, Tom, à un bouleversant retour du poète lui-même, gravement malade à son tour, d'une cloison de part et d'autre de laquelle on se guette, le souffle retenu, à une errance solitaire dans le blanc de la neige et le noir du deuil. Regards dans le regard, venant doubler le point de vue de Fanny de leur propre vision - dont nous ne saurons pas plus que ces brefs mais pénétrants coups d'œil de passagers clandestins à bord d'une histoire qui leur échappe et dont pourtant, nous le sentons, ils comprennent l'essentiel. Comme un scénario en germe au cœur du récit, il faudrait écrire une nouvelle fois toute l'histoire de ce point de vue d'enfance, de cette perspective dans la perspective, imaginer un nouveau Keats-et-Fanny sur la trame de ces regards croisés. Et y ajouter peut-être celui, tellement différent entre le début et la fin du film, de Brown - l'ami de Keats dont il partage un temps, dans la bourgade de Hampstead au nord de Londres, la moitié de la maison de Wentworth Place, la famille Brawne habitant l'autre -, ou celui, tout en mutations subtiles, de Mrs Brawne, mère aimante et figure maternelle de substitution pour Keats l'orphelin, peu à peu acquise à la difficile cause des amoureux, quand toutes les conventions, préventions et limitations matérielles de l'existence leur sont contraires. 
Par-dessus tous les non-dits, tous les indicibles, Jane Campion tisse la toile diaphane des regards, à mi-chemin du visible et de l'invisible, dans laquelle vient se prendre et se réfracter le réel, nous rappelant que la poésie n'est autre que cela : un regard intense sur le monde. Là où Keats pose son regard de poète sur la vie, celui de Fanny est habité d'un autre lyrisme : son amour même. Il transfigure ce qu'il touche à la manière du regard de poésie. La cinéaste se met à l'écoute de l'un et de l'autre - elle donne à voir ces visibles transfigurés, sous forme de visions, voire d'entrevisions, de fugitifs instants de miracle, de poésie pure. Lorsqu'au réveil, dans l'éclatante lumière du matin tamisée par les rideaux de sa chambre, Fanny, tout de blanc vêtue, passe de l'autre côté des voilages - avec un très léger bruissement du tissu : le film est entretissé de ces bruits infimes, joignant à la magie visuelle la poésie du presque imperceptible - pour regarder par la fenêtre, les mains appuyées sur les carreaux, on sent la charge d'amour qu'il y a soudain dans ce plan de caméra subjective révélant en plongée, de l'autre côté de la croisée, John allongé dans l'herbe un livre auprès de lui, qui se redresse un peu en l'apercevant, proche et lointaine. Temporalité flottante de ce furtif, cet infinitésimal événement amoureux où frémit un beau silence habité. Déjà la jeune fille - en plan rapproché serré, intimiste, comme nombre des cadrages de ce film -, visible de dos à travers le rideau beige en un lumineux jeu de transparences, se recule dans un mouvement à peine saisissable, les mains toujours appuyées contre la vitre. Instant de poétique incandescence, semblable, dans sa fulgurance, à une image échappée d'un poème, telle cette vision de Rilke :

Celle qu'on aime n'est jamais plus belle que lorsqu'on la voit apparaître encadrée de toi ; c'est, ô fenêtre, que tu la rends presque éternelle.

Tous les hasards sont abolis. L'être se tient au milieu de l'amour, avec ce peu d'espace autour dont on est maitre ${ }^{23}$.

\section{Art du mystère, mystère de l'art}

De telles visions, que Jane Campion laisse éclore puis s'effacer tout au long de Bright Star, sans insistance, avec la légèreté d'un nuage qui passe, l'évanescence d'une pensée, mais, dans le même temps, l'évidence d'un éblouissement

23. R. Maria Rilke, "Les Fenêtres», III, dans Vergers suivi d'Autres poèmes français, Paris, Gallimard, coll. «Poésie», 2005, p. I53. 
intérieur affleurant à la surface du visible, sont les propres images poétiques d'une cinéaste qui a souhaité inscrire la poésie de Keats non seulement dans la substance, mais dans la forme même du récit. Cette poésie, note-t-elle, "a inspiré toute la structure de l'histoire du film. Certains de ses poèmes ont été écrits sous forme d'odes, d'autres sous celle de ballades, et je me suis mise à réfléchir à l'histoire de Fanny et de John comme à une ballade, une sorte de poème narratif ${ }^{24}$ ", dans le style du long poème de Keats à l'atmosphère gothique, "La Veille de Sainte-Agnès" ("The Eve of St. Agnes»). Elle précise : "L'histoire progresse en strophes retraçant leur attachement de plus en plus profond et leurs difficultés de plus en plus grandes ${ }^{25}$.»

La séquence de Noël est particulièrement révélatrice de la manière dont Jane Campion intègre à son récit la poésie keatsienne, lui accorde une place concrète, et, dans le même temps, compose son "poème narratif" cinématographique, en y faisant la part belle à l'imagination, à l'ellipse, au mystère, à l'imperceptible, au clair-obscur, au silence constellé de regards et de mots vrais, du cœur ou du poème. Keats s'est joint à la famille Brawne pour le réveillon et, dans la lumière douce du feu de cheminée, la lueur mordorée des chandelles se reflétant sur les verres et dans les yeux, l'harmonie d'un soir de Noël dont Fanny dira elle-même plus tard qu'il fut «le jour le plus heureux qu'il [lui] avait été donné de vivre jusque-là26", quelque chose se noue, mezzo voce. Instants de complicité grandissante, cristallisés en plans rapprochés, hasards qui n'en sont pas : Fanny fait remarquer à John que le lit du poète dans la maison de Wentworth Place se trouve être celui qu'elle avait occupé l'été précédent, lorsque sa famille avait loué l'aile de Mr Brown et de Keats, en leur absence. Pour preuve, et comme un signe annonciateur, cette petite silhouette gravée par la jeune fille sur le mur, près de l'oreiller : une princesse de conte de fées, aux ailes de papillon... Ce double détail, à l'instar de nombreux autres, a été imaginé par la réalisatrice afin de faire exister poétiquement les intervalles, les espaces vides entre les données consignées. «Le film reste le plus fidèle possible aux faits historiques. Il m'a fallu cependant inventer ce qui se passait entre les faits connus et répertoriés. Ma démarche a été de rester la plus modeste possible, et la plus proche de l'esprit de ces deux êtres extraordinaires ${ }^{27}$ ", explique-telle. Ainsi, en ce qui concerne ces détails - romantiques - du lit et du mur, qui seront appelés à jouer un rôle essentiel dans la composition poétique du film, elle précise :

24. J. Campion, dans dossier de presse cité, p. 6 ; je souligne.

25. Ibid., p. 4.

26. "[...] "the happiest day I had ever then spent" [...]» (cité par A. Motion, Keats, ouvr. cité, p. 334 ; je traduis).

27. J. Campion, dans dossier de presse cité, p. 6. 
En réfléchissant à tous les aspects pratiques de leur relation et de leurs vies, j'ai réalisé qu'il était possible que Fanny ait pu de fait dormir dans ce qui devait devenir le lit de Keats lorsque lui et son meilleur ami Brown étaient en voyage en Écosse. De même, quand la famille de Fanny a déménagé pour partager la maison avec Brown et Keats, Fanny et Keats ont pu ne dormir qu'à une cloison de distance ${ }^{28}$.

Il s'agit en quelque sorte d'une mise en œuvre du principe keatsien par excellence de la capacité négative, une manière de laisser rayonner la poésie dans tous les espaces du film - et la poésie, ici, plus que jamais, c'est la vérité, c'est la beauté, c'est la réalité supérieure exaltée par Keats lui-même, ou Novalis. Évoquant tour à tour la retenue dont faisait habituellement preuve Fanny - et qui imprègne la narration elle-même - et sa propre quête de cinéaste, Campion résume : "La vérité se trouvait quelque part entre tous ces contrastes, entre ces effusions passionnées, cette douleur et cette réserve extraordinaire, tout ce qui façonnait le monde dans lequel ils ont affronté leur destin ${ }^{29}$. " Ainsi, la simple évocation de la petite silhouette de princesse aux ailes de papillon permet de tisser des liens d'évidence, à forte charge poétique, avec d'une part la séquence des papillons - imaginée à partir d'une lettre de Keats, et qui deviendra un symbole puissant de l'amour unissant Fanny et John -, et d'autre part un poème de Keats, l' "Ode à Psyché» - Psyché, ou l'âme, a souvent été représentée avec des ailes de papillon - : "Sûrement ai-je rêvé aujourd'hui, ou ai-je vu / L'ailée Psyché de mes yeux éveillés ${ }^{30}$ ?"

D’allusions subtiles en entretissages délicats des éléments biographiques et de l'œuvre, la poétique de Jane Campion repose sur une approche intuitive et métaphorique, une réappropriation profonde de la capacité négative. Keats lui-même ne constatait-il pas : "La vie d'un Homme de quelque valeur est une allégorie continuelle - et très peu d'yeux savent voir le Mystère de sa vie - une vie comme les Écritures, figurative ${ }^{3 \mathrm{I}}$ »? Les yeux de la réalisatrice sont de ceux qui savent voir ce mystère-là, en rendre sensible, partageable l'infinie poésie. Dans cette même séquence de Noël, à la demande générale, Keats se met à réciter son poème "When I have fears that I may cease to be..." ("Quand je crains de cesser

28. "By thinking about all the practical aspects of their relationship and their lives I realised it was possible that Fanny may have actually slept in what was to become Keats's bed while he and his best friend Brown were away in Scotland. Also when Fanny's family moved in to share the house with Brown and Keats, Fanny and Keats may have slept only a wall apart." (J. Campion, "My John Keats", dans "Bright Star": The Complete Poems and Selected Letters, ouvr. cité, p. xv; je traduis.)

29. J. Campion, dans dossier de presse cité, p. 6; je souligne.

30. J. Keats, Poèmes et Poésies, ouvr. cité, p. I8I.

3I. "A Man's life of any worth is a continual allegory — and very few eyes can see the Mystery of his life-a life like the scriptures, figurative [...]» (J. Keats, cité dans A. Motion, Keats, ouvr. cité, p. 355 ; je traduis). 
d'être...»), marqué comme toute son œuvre par le sentiment aigu de la précarité d'être, l'inquiétude de la disparition, la proximité de la mort - et ce n'est pas là une manière artificielle de faire apparaitre sa poésie, mais un acte poétique en soi, venant en quelque sorte, dans son mode de surgissement imaginé, au fil d'une soirée de Noël où affleurent tant d'éloquents non-dits, confirmer celle qui est donnée à entendre, l'incarner au cœur de la vie même, conférant à ce surgissement une dimension de nécessité intérieure, là où la citation aurait pu rester arbitraire.

Le passage suivant du sonnet est particulièrement significatif en ce sens :

Lorsque j'aperçois, sur la face étoilée de la nuit,

Les immenses symboles nuageux d'une haute romance,

Et pense que peut-être je ne vivrai jamais pour tracer

Leurs ombres, avec la main magique du hasard ${ }^{32}[\ldots]$.

En effet, lorsque le poète parvient au vers «Huge cloudy symbols of a high romance» ("Les immenses symboles nuageux d'une haute romance»), il regarde Fanny, et ne peut poursuivre, en proie à un trouble soudain dont, à l'instar de la jeune fille, manifestement bouleversée, nous devinons le sens et la profondeur. Campion accentue ici la connotation amoureuse, en jouant sur le double sens anglo-saxon de "romance» : épopée, récit souvent basé sur des amours chevaleresques; et histoire d'amour. Et «sur la face étoilée de la nuit» de Noël, nous lisons, en même temps que les protagonistes, le haut amour en train de naître et qui - mise en abyme - deviendra récit, poème narratif, film empreint d'une même lumière. Le mur, le lit, la princesse ailée, la nuit, l'étoile, un chat noir et blanc, Topper, auquel tantôt l'un, tantôt l'autre prodigue des caresses, sorte d'esprit familier établissant un lien secret, inconscient entre eux, apparaissent dès lors comme autant de correspondances subtiles. Tout un réseau de liens, de symboles, de signes, de présages à peine esquissés se tisse. C'est, parfois, une musique qui revient, comme une réminiscence prenant soudain tout son sens. $\mathrm{Ou}$, ailleurs, l'éclair sombre d'une portière qui claque : le poète en partance pour l'Italie, qui vient de faire ses adieux à Fanny et, les yeux perdus dans le vague, n'est plus que l'ombre de lui-même sur la banquette de la diligence. Comme un clap de fin, un tombeau que l'on scelle - et soudain l'on sent remonter le souffle froid du vide, depuis les profondeurs d'un gouffre intérieur insondable. Cinéma de ressentis, d'intuitions, non de démonstrations : comme la poésie elle-même. À l'instant même où cette portière se referme sur la vie de Keats, s'ouvre un paysage de collines embrumées aux tonalités gris-brun, où passent ensemble à tire-d'aile deux oiseaux solitaires, précédés et accompagnés de

32. "When I behold, upon the night's starr'd face, I Huge cloudy symbols of a high romance, I And think that I may never live to trace / Their shadows, with the magic hand of chance [...]" (J. Keats, "Bright Star": The Complete Poems and Selected Letters, ouvr. cité, p. $30 \mathrm{O}$; je traduis). 
leur appel strident, mélancolique. Fulgurance d'un vol traversant le champ de droite à gauche comme un souvenir, un présage, une éloquente entrevision semblables à ceux qui parcourent en filigrane Bright Star, à l'instar de l'œuvre du poète elle-même, et auxquels il était attentif.

Ce langage subliminal, métaphorique, est celui de l'amour : par lui les âmes éprises se comprennent à demi-mot, communiquent, en lui elles se retrouvent, dans une solitude sacrée - "Ô Solitude! [...] / [...] et sûrement ce doit être / Presque la plus haute félicité du genre humain, / Lorsque vers tes retraites s'enfuient deux âmes sœurs ${ }^{33}$.» Dans la très brève parenthèse où ils resteront seuls - mais avec l'œil de Samuel, certes en retrait, rangeant son violon, tout de même posé sur eux -, John prend doucement la main de Fanny. Temps suspendu, infimes et éternels instants d'émotion, de silence vibrant. La poésie, plus que jamais, réside ici dans le non-dit autant que dans le verbe, dans le révélé autant que dans l'invisible : dans cette union intime des deux qu'est le mystère. «La poésie est une expérience des sens. On ne plonge pas dans un lac pour regagner aussitôt la berge, mais pour être dans le lac, pour s'abandonner à la sensation de l'eau. On ne tente pas de comprendre le lac, c'est une expérience qui dépasse la pensée. La poésie apaise et invite l'âme à accepter le mystère", dit le Keats de Campion, toujours en harmonie avec la capacité négative, au cours d'une "leçon de poésie» qu'est venue prendre Fanny; ce à quoi la jeune fille répond : "J'aime le mystère.» La cinéaste a pris soin de préserver celui-ci, dans un accord parfait avec le credo keatsien, tout au long du film :

Il était important pour moi de cultiver le mystère de cette relation exceptionnelle, ne pas tout dévoiler, laisser vivre ces personnages au milieu d'une nature qui respire la générosité et la sensualité au fil des saisons. Filmer un arbre, écouter le souffle du vent sur des champs de fleurs, observer le vol de papillons dans une chambre, s'attacher à un baiser ${ }^{34}$.

\section{«Un chant d'amour trop doux pour les lyres terrestres ${ }^{35}$ »}

À la vue d'arbres en fleurs sur une colline de Rome, Keats, peu de temps avant sa mort, dira à Severn, l'ami peintre qui l'a accompagné jusqu’à la fin en Italie : «Le printemps a toujours été un enchantement pour moi... peut-être

33. "O Solitude! [...] / [...] and it sure must be /Almost the highest bliss of human-kind, / When to thy haunts two kindred spirits flee." (J. Keats, "O Solitude! if I must with thee dwell" ["Ô Solitude, si je dois avec toi demeurer...»], dans "Bright Star": The Complete Poems and Selected Letters, ouvr. cité, p. $4 \mathrm{I}$; je traduis.)

34. J. Campion, "Un amour incandescent d'un romantisme absolu», art. cité.

35. "A song of love, too sweet for earthly lyres» (J. Keats "Lamia», dans "Bright Star": The Complete Poems and Selected Letters, ouvr. cité, p. I95; je traduis). 
le seul bonheur que j'aie eu en ce monde - aura été la croissance silencieuse des fleurs ${ }^{36}$." Jane Campion se met à l'écoute de ces floraisons muettes, de ces silhouettes d'arbres qui, dans le vert éclatant de leurs feuillages neufs, les couleurs tendres de leurs fleurs, ou le dénuement de leurs branches livrées à la neige, scandent le passage des saisons et du temps. En ce printemps I8I9, à Hampstead, amoureux de Fanny et habitant tout près d'elle dans l'autre aile de la maison de Wentworth Place où elle vit désormais, Keats, en un extraordinaire élan de créativité au cœur d'une nature à l'unisson de ses sentiments, compose parmi les plus belles pages de sa poésie - dont l'envoûtante «Ode à un rossignol» ("Ode to a Nightingale»). Le moment de la naissance de cette dernière - instants de grâce - est saisi dans toute son intensité par Jane Campion. Cette séquence - et celle qui la suit immédiatement, de la mise au propre des feuillets dans un travail commun avec Brown - est directement inspirée d'un passage de la biographie de Motion, où figure le compte-rendu fait par Brown de la genèse du poème :

Au printemps de I8I9, un rossignol avait construit son nid près de ma maison. Keats ressentait une joie tranquille et continuelle dans son chant; et un beau matin il prit une chaise de la table du petit-déjeuner pour l'emporter jusqu'au gazon sous un prunier, où il resta assis deux ou trois heures durant ${ }^{37}$.

Ici, c'est de nouveau de la perspective de Fanny, tout d'abord selon un angle de caméra subjective, qu'est vue la scène. La jeune fille, assise sur une chaise à l'intérieur, est à son ouvrage, et regarde par la porte-fenêtre, sur une partie de laquelle joue - élément et mouvement récurrents - un rideau clair qu'agite la brise printanière. L'arbre se dresse devant la fenêtre, dans la lumineuse profusion de sa floraison. Fanny est en robe bleu clair, avec dans la silhouette quelque chose d'une peinture hollandaise, ou du romantisme pictural d'un Caspar David Friedrich. Keats entre dans le champ, une chaise à la main, et va s'asseoir au pied de l'arbre. Chants d'oiseaux, et silence. Souffle de la brise, toujours si palpable - souffle de la plénitude. Coup d'œil de Fanny par la fenêtre : le contrechamp dévoile, en l'une de ces éblouissantes visions ponctuant de leur surgissement le poème narratif, un cadre constitué par les branches fleuries parmi lesquelles, dans la radieuse lumière, jouent des ombres au rythme de la brise - et, de l'autre côté de celui-ci, le jeune homme plongé dans ses pensées.

36. "The spring was always enchantment to me... perhaps the only happiness I have had in the world - has been the silent growth of flowers." (Cité dans A. Motion, Keats, ouvr. cité, p. 564; je traduis.)

37. "In the spring of I8I9 a nightingale had built her nest near my house. Keats felt a tranquil and continual joy in her song; and one morning he took a chair from the breakfast table to the grass-plot under a plum-tree, where he sat for two or three hours." (Ibid., p. 394-395; je traduis.) 
Élément éminemment poétique, sa voix off - sa voix intérieure de poète à l'instant de la création - s'élève sur les premiers mots du poème : "My heart aches..." ("Mon cœur souffre...»). La première strophe se développe peu à peu, vie et mort entremêlées comme toujours chez Keats, accompagnée par une série de cadrages sur le poète, le révélant tantôt de face, en plan rapproché serré, tantôt de plus loin, de dos sur sa chaise, sous cet arbre en fleurs qui rappelle soudain ceux de $\mathrm{La} \mathrm{Cerisaie}^{38}$, et leur paradoxal message si omniprésent dans la poésie japonaise, comme chez Kobayashi Issa : «Prépare-toi à la mort / prépare-toi / bruissent les cerisiers en fleurs ${ }^{39}$ ".

Campion, en captant dans toute sa fragile splendeur cette nature qui donne naissance à l'œuvre d'art, en quelque sorte à sa source, au moment privilégié de l'inspiration, de la création, entremêle - élément caractéristique et récurrent de sa poétique filmique - «réel» et poésie, un réel interagissant avec la poésie, la nourrissant, et déjà modifié par elle - transfiguré. Dans cette saisissante vision, la poésie apparaît soudain comme une victoire - certes relative - sur l'éphémère et la mort qui rôde, en même temps que célébration de cet éphémère. Guidée par la main en très gros plan du poète, la plume glisse sur le papier posé sur un livre, surface où se poursuit au gré du souffle d'air le jeu léger de l'ombre et de la lumière, toujours accompagné en arrière-plan sonore des chants d'oiseaux, et de la voix off disant le poème au premier plan. Mais un autre coup d'œil de Fanny révèle fugitivement la chaise à présent vide sous l'arbre en fleurs. Un bref instant, la jeune fille semble chercher du regard celui qu'elle aime - et c'est là comme une vision subliminale prémonitoire, une anticipation furtive sur le destin, sur l'absence : celle, relative, qui va être annoncée tout de suite après, le poète s'éloignant pour un temps de Hampstead ; mais surtout l'absence définitive, l'absence absolue de la mort à venir de Keats, ailleurs, dans une autre saison.

Dans la séquence suivante, Keats et Brown reconstituent et recopient le travail qui vient de naître, dont Fanny, depuis l'obscurité du couloir d'abord - mise en abyme toujours entrelaçant vie des personnages et poésie -, puis passant avec grâce de l'ombre à la lumière de la pièce, entend la puissante sixième strophe :

Plongé dans l'obscurité, j'écoute; et plus d'une fois J'ai été presque amoureux de la Mort apaisante,

38. Voir en ce sens le superbe traitement cinématographique de la pièce de Tchékhov et de ses efflorescences par M. Cacoyannis (1999), évoqué dans mon étude "La Cerisaie, de M. Cacoyannis : barcarolle russe du monde flottant», dans CinémAction, "Tchékhov à l'écran ", M. Estève et A. Z. Labarrère (dir.), Condé-sur-Noireau, Éditions Charles Corlet, 2013, p. I64-I75. 39. K. Issa, dans Haiku. Anthologie du poème court japonais, Paris, Gallimard, coll. "Poésie», 2004, p. 60. 
Lui ai donné de doux noms en maints vers songeurs,

Pour qu'elle emporte dans l'air mon souffle calme;

Maintenant plus que jamais il semble magnifique de mourir,

De cesser d'être à minuit sans souffrance,

Tandis que tu épanches ton âme aux quatre vents

Dans une telle extase ${ }^{40}$ !

On retrouvera l' "Ode à un rossignol» dans son intégralité en voix off, sur le fond noir du générique final, le deuil consommé, accompagnée de l'adagio de la Sérénade en si bémol majeur, K. 36I de Mozart (dite "Gran Partita»), conçue pour douze instruments à vent et contrebasse, mais interprétée ici dans une version chorale arrangée par Mark Bradshaw (Human Orchestra), sur laquelle se détache la voix du soliste, qui n'est autre que le poète lui-même, dans la scène du concert. Les dernières mesures correspondront alors, avec une évidence symbolique et poétique, au tout derniers vers de l'ode : "Fled is that music:-do I wake or sleep?» ("Cette musique s'est envolée : - suis-je éveillé, ou bien endormi?»). Ce subtil et bouleversant adagio de Mozart, revenant à des moments clés mais, comme toujours chez Campion, sans aucune insistance, est interprété lors d'un concert d'hiver a capella donné par les habitants de Hampstead, un chant choral réunissant des voix masculines parmi lesquelles se trouve, haute, cristalline, celle de Keats. Les membres de la chorale sont assis au centre de la pièce, entourés de leur auditoire. Fanny se tient debout dans un coin et observe : en contrechamp et cadré plus serré, dans son exact angle de vue apparaît, telle une entrevision, le poète. Le regard amoureux détoure l'être sur le fond de ce et de ceux qui l'entourent. Il révèle, mais avec une infinie retenue, presque à son insu, un lien en train de naître. Comme s'il sentait ce regard de la jeune fille sur lui, comme s'il la cherchait aussi presque inconsciemment des yeux, il fixe très fugitivement la caméra - et, dans cette atmosphère si particulière, si feutrée, si intimiste, nimbée de la lumière douce des chandelles, on sait que quelque chose d'infime et d'immense, ténu mais palpable, s'est définitivement scellé entre ces deux êtres. La poésie est faite de cette certitude lumineuse, mais à peine effleurée, et la vision est toujours, dans sa fulgurance secrète, son mode d'apparition privilégié. Comme si la vérité et la beauté profondes des êtres ne pouvaient se révéler que par éclairs, dont le cinéma serait alors parfois le témoin inespéré.

40. "Darkling I listen; and for many a timelI have been half in love with easeful Death, / Call'd him soft names in many a mused rhyme, / To take into the air my quiet breath; / Now more than ever seems it rich to die, I To cease upon the midnight with no pain, / While thou art pouring forth thy soul abroad/In such an ecstasy!» (J. Keats, "Bright Star": The Complete Poems and Selected Letters, ouvr. cité, p. 237; je traduis.) 


\section{«Rapide comme le passage d'une larme d'ange ${ }^{4 \mathrm{I}}{ }$.}

Bright Star est comme enchâssé entre deux visions jumelles, qui en constituent les deux pôles, et le placent d'emblée dans la perspective de Fanny. La première, très claire, dédiée à la lumière, ouvre le film : en une succession de magnifiques très gros plans, un fil blanc passe dans le chas d'une aiguille, puis l'aiguille elle-même passe à travers une étoffe blanche - mouvement familier et pourtant si mystérieux. Le plan suivant révélera la jeune fille cousant à sa fenêtre dans le matin neuf - essentielles fenêtres, dans leurs modulations de la lumière du jour ${ }^{42}$ où, penchée sur un ouvrage ou un livre, vient souvent se découper, de profil, une silhouette à la Vermeer. La musique qui accompagne cette danse de la lumière au plus près de la matière est, déjà, cet adagio épuré de la Sérénade en si bémol majeur de Mozart, qui sera donc entendu lors du concert a capella, mais où pour cette fois, se juxtaposant aux voix aériennes, frémissent des cordes profondes. La présence dès ce début de film de cette musique récurrente - musique dont on saisira la portée, dans le sens d'une boucle bouclée, d'une sorte de révélation, au moment du concert et de l'entrée dans le chant et le champ de John en tant que soliste - apparaît ici encore comme un signe annonciateur du lien à naître, et qui est déjà là en substance, dans cette voix chantée du poète se superposant au geste de couture de Fanny. Musique-prémices, musique-leitmotiv amoureux, imprimant dès les premiers instants un élan secret, une poétique direction.

La seconde vision, tel un négatif de celle qui illumine les premières images du film, se situe à la fin de celui-ci, lorsque le fil des jours a sombré dans la mort et l'obscurité. Plus de musique, mais un silence sépulcral surplombant cet autre très gros plan sur une étoffe. Ce n'est plus cette fois le tissu clair d'une robe de fête que l'on coud, mais la doublure d'un vêtement de deuil que parcourt de points noirs la même main. Image double, positif-négatif. Là, l'espoir, la page blanche ouverte sur la vie. Ici, la mort, le livre qu'on referme. Les deux bornes de l'existence - celle si brève d'un poète disparu à vingt-cinq ans -, les deux pôles d'une œuvre. «J'ai deux voluptés à méditer lors de mes promenades,

4I. "E'en like the passage of an angel's tear" (J. Keats, "To one who has been long in city pent" ["À qui est resté longtemps confiné dans la ville»], dans "Bright Star": The Complete Poems and Selected Letters, ouvr. cité, p. 42 ; je traduis.)

42. Cette fascinante utilisation de la lumière, des fenêtres, rideaux et autres éléments du passage ainsi que cette picturalité ne sont pas sans rappeler celles d'un Rohmer dans La Marquise d'O... (1976) d'après Kleist. Voir à ce sujet mon étude : «Une danse d'ombre et de lumière au bord du gouffre (La Marquise d'O...) ", dans Rohmer ou le jeu des variations, P. Louguet (dir.), Saint-Denis, PUV, coll. «Esthétiques hors cadre», 20I2, p. I59-I89. 
votre Beauté et l'heure de ma mort. Ô si je pouvais les posséder toutes deux à la même minute ${ }^{43}$ ", écrivait Keats à Fanny. Dans ce geste-mémoire final d'une aube triste - juste avant, en un accomplissement rituel, de se couper les cheveux -, beauté et mort se trouvent réunies. Sur la table, pêle-mêle, les livres donnés par le poète, et puis ses lettres éparses, lues et relues toute la nuit, comme elles le seront souvent au fil des années qui suivront, dans la solitude endeuillée d'une chambre.

Une autre image double résonne longuement au cœur du film, une autre double vision habitée de vie et de mort, par la fenêtre du salon que Keats partage avec son ami Brown, dans l'une des deux ailes de Wentworth Place, les Brawne occupant l'autre. Dans l'avant-printemps de I820, la voix off de John dit les phrases de l'une des lettres écrites à Fanny au cours des semaines où il doit rester confiné à la maison en raison de sa maladie : «Ma tendre créature, / Quand j'expédierai ceci, je serai dans le salon, espérant vous apercevoir un instant dans le jardin.» C'est alors que, comme une vision intérieure s'incarnant, une anticipation émotionnelle, une sorte de flash forward, la réalisation sous nos yeux de son rêve en somme, apparaît Fanny dans le cadre de cette fenêtre, dans ce jardin où jouent Toots et Sam : appuyée contre un grand arbre, le vent dansant dans sa jupe, le visage tourné dans la direction de John. Cette scène dans le jardin commun est bien vue de la perspective du poète, cette fois. Fanny s'approche de la fenêtre - un même plan réunit les amoureux, de part et d'autre de la mince paroi de verre. Montrant un petit papier plié et une plume, elle embrasse le billet. Chassée par Brown, qui voit d'un mauvais œil cet attachement, elle recule, mais ne cède point : elle envoie baisers et sourires à John. Instants d'échange amoureux, heureux, se jouant de tous les obstacles.

C'est une tout autre atmosphère qui règne dans la scène qui constitue le pendant et le négatif de celle-ci, peu avant la fin du film. Keats est parti pour l'Italie dont il ne reviendra pas. C'est un entre-deux, dont l'issue n'est que trop prévisible. Dans cette même pièce qu'il partageait avec son ami, Brown se tient désormais seul, songeur. Son regard est attiré par une image qui se reflète dans le miroir accroché au mur : on entend des rires d'enfants, un peu étouffés par la neige, Sam et Toots jouent dans le jardin, font une bataille de boules de neige - ce reflet est celui de la fenêtre. Cette même fenêtre par laquelle, en une lointaine belle saison, Keats regardait sa bien-aimée au-dehors, s'attirant les foudres de Brown. Cette fenêtre dans le miroir dessine un pur cristal-temps deleuzien : nappes de passé, pointes de présent, les strates temporelles s'entremêlent, se superposent, réveillant l'indicible. Toute la sensation de l'éphémère, du révolu, la profondeur de la nostalgie, de l'absence, la conscience des saisons

\section{J. Keats, Lettres à Fanny et autres correspondances, ouvr. cité, p. 422.}


qui passent - celles du dehors, et celles intérieures : alliance puissamment romantique, comme dans le poème de Keats "Les saisons humaines» ("The Human Seasons") -, tout ce sentiment du temps est cristallisé dans ce reflet du miroir et dans la scène suivante. Brown en effet se retourne et regarde à présent par la «vraie» fenêtre. Il s'assoit, et observe avec gravité le spectacle dans le jardin hivernal. De nouveau, ce qui nous est donné à voir ressortit à un angle de caméra subjective - cependant son regard a remplacé celui de Keats. Arbres, neige, jeux d'enfants : mais l'innocence est perdue. Si Toots et Sam courent en tous sens, continuant leur joyeuse bataille, Fanny fait simplement mine d'y participer, son âme s'est retirée de ses gestes. De l'autre côté de la croisée, elle traverse lentement le champ, silhouette sombre dans tout ce blanc, tête baissée, l'air absent, une boule de neige dans la main, dont elle ne fait rien. Un plan rapproché de Brown cache mal la légère mélancolie de son visage, et tout ce qui se passe probablement en lui. Le souvenir du passé, de la présence de John posant le regard sur Fanny par la même fenêtre - «Les choses, regarde-les aussi avec les yeux de ceux qui ne les voient plus ${ }^{44}$ », invitait Pirandello, et Brown semble répondre à cet appel, en cet instant silencieux. Ses regrets aussi, sans doute, de n'avoir pas su vraiment voir, ni su être là pour son ami lorsque, à l'heure du voyage lointain, il aurait eu besoin de son soutien. Sa compréhension tardive et sa compassion aussi, peut-être, pour ce que vit cette jeune fille triste de l'autre côté de la vitre. Un nouveau plan la montre debout, face à la fenêtre, elle s'est immobilisée, la boule de neige toujours dans la main, le regard dans le vague : soudain elle tourne la tête, regarde face caméra, vers la fenêtre, les traits pleins de mélancolie. Que voit-elle - Brown? Partagent-ils alors un peu de la même souffrance, de la même nostalgie, dans cet étrange entre-deux, ce mutique face-à-face de part et d'autre d'une vitre? Ou bien revoit-elle tout ce qui a disparu, de cette disparition si keatsienne - «Le jour s'est enfui, et toutes ses douceurs se sont enfuies! / [...] / Disparus hors de saison à la tombée du soir ${ }^{45}$ »-, revoit-elle le fantôme de son amour, des beaux jours dans la brise et les baisers envoyés par la fenêtre? Son regard ne s'attarde pas, rien ne s'attarde ici, tout s'écoule dans l'élan de ces strophes filmiques, l'enchaînement de ces ellipses denses où les trop-pleins s'écrivent en creux. Mais la vision, elle, reste, rémanence infiniment poétique, contrepoids de toute perte.

44. L. Pirandello, «Colloques avec des personnages», dans Nouvelles complètes, Paris, Gallimard, coll. "Quarto", 2000, p. 2I22. Voir cette séquence, filmée de manière bouleversante par V. et P. Taviani, dans l'épilogue de Kaos, "Colloquio con la madre" (1984).

45. "The day is gone, and all its sweets are gone! /[...] / Vanished unseasonably at shut of eve [...]" (J. Keats, "The day is gone» ["Le jour s'est enfui...»], dans "Bright Star": The Complete Poems and Selected Letters, ouvr. cité, p. 36I; je traduis). 


\section{Passages secrets}

D'autres fenêtres s'ouvrent, ailleurs, sur la plénitude et la beauté d'être. Juste après leur premier baiser, Fanny et Keats s'en reviennent radieux, marchant derrière Toots dans la complicité d'une nature luxuriante où chemin faisant, parmi les arbres éclatants de fleurs, ils se mettent à jouer à «un, deux, trois, soleil», s'embrassant à la dérobée, se tenant et se lâchant la main, courant follement et s'immobilisant à chaque fois que la petite fille, qui se prend à leur amoureuse espièglerie, se retourne, improvisant un enfantin et délicieux hymne au bonheur. Des entraves de la vie - être constamment sous le regard et la surveillance d'autrui -, ils font un moment de poésie, recueilli en sa douce allégresse par Jane Campion, dans l'accompagnement cristallin des cordes qui se poursuit, en transition sonore, sur le plan suivant, une vision passant tel un souffle : au terme de ce retour plein d'euphorique légèreté, Fanny est allongée les yeux clos sur son lit clair, face à la fenêtre ouverte, dans une lumière éblouissante et tendre, presque irréelle. Au rythme de la brise tiède qui module par bouffées l'espace de sa chambre, des ombres évanescentes dansent sur le mur, ainsi qu'en rêve, comme dans l'"Ode sur l'indolence»: "Mon sommeil avait été brodé de rêves indécis; / Mon âme avait été une clairière parsemée / De fleurs, et d'ombres mouvantes [...] : / [...] / La fenêtre ouverte pressait une vigne aux feuilles nouvelles, / Laissait entrer la chaleur naissante et le chant de la grive musicienne ${ }^{46}[\ldots]$.» Comme en écho à ce poème, le fameux chant du rossignol s'élèvera dans la continuité de cette brève vision d'une ardente poésie, intimement reliée à tant d'autres parcourant les poèmes de Keats : «Ce même chant qui bien des fois / A charmé des fenêtres magiques, s'ouvrant sur l'écume / De mers périlleuses, dans des royaumes de fées oubliés77 ( "Ode à un rossignol»); "et une fenêtre ouverte dans la nuit, / pour laisser entrer le fervent Amour ${ }^{4}$ !» ("Ode à Psyché»)... C’est bien un vent de passion qui souffle désormais dans l'univers de Fanny, un vent présent dans la poésie de Keats, ainsi dans l'"Ode à Psyché» toujours : «En quelque région inexplorée de mon esprit, / Où des pensées aux rameaux nouvellement nés d'une douce

46. "My sleep had been embroider'd with dim dreams; / My soul had been a lawn besprinkled o'er / With flowers, and stirring shades [...]: / [...] / The open casement press'd a new-leaved vine, / Let in the budding warmth and throstle's lay [...]" (J. Keats, "Bright Star": The Complete Poems and Selected Letters, ouvr. cité, p. 359; je traduis).

47. "The same that oft-times hath / Charm'd magic casements, opening on the foam / Ofperilous seas, in faery lands forlorn." (Ibid., p. 238; je traduis.)

48. "[...] and a casement ope at night, / To let the warm Love in!» (ibid., p. 242 ; je traduis). 
douleur / À la place des pins murmureront dans le vent ${ }^{49}$ ». Dans un contexte amoureux, ce vent se fera parfois véhément, comme dans «La Veille de SainteAgnès»: “"Écoute! C’est une tempête d'elfes venue du pays des fées [...]”. / [...] / Et ils s'en allèrent : oui, il y a des éternités / Que ces amants se sont enfuis dans la tempête ${ }^{50}$.» Une tempête qui n'est pas sans rappeler celle qui fait rage chez Dante, dans ce chant $V$ de l'Enfer si essentiel, on le verra, pour Keats, où Dante, désignant à son guide le couple d'amoureux damnés formé par Paolo et Francesca, lui dit : «Poète, volontiers / je parlerais à ces deux-ci qui vont ensemble, / et qui semblent si légers dans le vent ${ }^{51}$." Mais c'est un vent doux qui se lève plus d'une fois dans Bright Star, et tout particulièrement dans cette scène où, alliée à la lumière vibrante, il emplit la pièce d'une atmosphère onirique, tout à la fois éthérée et sensuelle : si keatsienne. Il s'empare voluptueusement des matières, soulevant par vagues successives le rideau de voile blanc qui s'envole, diaphane - comme en écho à «l'amour aux rideaux parfumés ${ }^{5^{2}}$ » chanté par le poète -, jouant avec le tissu léger de la longue jupe de Fanny, souffle suave auquel elle s'abandonne, comme en prière, paumes ouvertes, extatique. Puissance contemplative d'une telle image-rêve, «en alliance intime / Avec les esprits de l'air et les vastes visions ${ }^{53}$ » de Keats.

À l'instar de cette scène à l'unisson romantique des éléments et de l'âme de l'autre, une communication amoureuse sans mots s'établit entre les jeunes gens à travers la cloison qui les sépare désormais, après l'emménagement de la famille Brawne dans l'autre aile de la maison de Wentworth Place. Séquence d'un lyrisme saisissant mais retenu, baignée d'une clarté révélatrice et sublime, comme une mise en lumière des sentiments. John, qui est monté dans sa chambre, entend les pas de Fanny dans l'escalier, puis dans la pièce d'à côté. Se dirigeant alors vers le mur, il y appuie ses mains et son front - puis tape deux petits coups contre la paroi. De l'autre côté, Fanny à son tour s'élance vers celle-ci, y pose la main. Une succession de champs-contrechamps d'une absolue poésie réunit doucement, en plans rapprochés serrés, les amoureux séparés par la mince cloison blanche dans laquelle se reflètent leurs profils - ombres, dédoublements faisant de cette surface qui à la fois scinde et rapproche un

49. "In some untrodden region of my mind, / Where branchèd thoughts, new grown with pleasant pain, / Instead of pines shall murmur in the wind [...]" (ibid., p. 24I; je traduis).

50. " "Hark!'tis an elfin storm from faery land [...]". / [...] / And they are gone: ay, ages long ago / These lovers fled away into the storm." (Ibid., p. 234-235; je traduis.)

5I. Dante, La Divine comédie. L'Enfer, éd. bilingue, trad. J. Risset, Paris, Flammarion, coll. "GF", I992, p. 63.

52. "fragrant-curtain'd love» (J. Keats, "The day is gone» [ "Le jour s'est enfui...»], dans "Bright Star": The Complete Poems and Selected Letters, ouvr. cité, p. 36I ; je traduis).

53. "La Veille de Sainte-Agnès», dans J. Keats, Poèmes et poésies, ouvr. cité, p. I63. 
miroir à traverser par la pensée, l'esquisse du geste, les battements de cour. Fanny répond de deux coups légers, puis retient son souffle. Le poète, en l'entendant, ferme les yeux, submergé par une vague d'émotion. Un insaisissable frémissement parcourt ses doigts. De l'autre côté, l'oreille et la main contre la cloison, Fanny attend. Son silencieux interlocuteur presse et passe doucement la main sur la surface claire. Alors la jeune fille, comme si elle sentait cette imperceptible caresse, effleure à son tour le mur et reste les mains appuyées sur celui-ci, regardant troublée devant elle. John et Fanny se tiennent ainsi de part et d'autre de la mince séparation, les mains invisiblement posées l'une contre l'autre, souffle et temps suspendus dans le silence et la presque immobilité, ouvrant un passage secret dans l'infranchissable même. John déplace ensuite son divan contre le mur - geste que Fanny reproduira de son côté, dans une autre scène. Cette traversée tendre des apparences, de l'opacité du réel, se renouvellera plus tard, dans cette même ferveur de la présence-absence, ce partage du geste tout en délicatesse et sensualité.

\section{«sur les ailes invisibles de la Poésie ${ }^{54}$ »}

Tout au long de Bright Star s'égrènent d'autres visions, entremêlant le rêve parfois éveillé, la poésie de Keats et la vie. Telle cette séquence d'été où Fanny, après une longue et douloureuse attente, reçoit la première lettre de John, de Shanklin, sur l'île de Wight, qui voit l'émergence tour à tour de plusieurs de ces éblouissements poétiques. Tandis qu'elle lit cette lettre à la fenêtre, silhouette claire près des rideaux plus sombres, nimbée de lumière, touchée par la grâce de l'amour, et que la voix off de Keats en prononce les mots - Jane Campion, ici comme ailleurs, opère un montage/remontage du matériau constitué par la correspondance, modifiant l'ordre de certains passages, en raccourcissant d'autres -, se dessine le visage du poète, en plan rapproché serré, de trois-quarts, le regard perdu dans le lointain, comme si l'écho de ses phrases en sa lectrice, sous l'effet d'une muette incantation, l'avait fait apparaître, l'avait appelé à la surface de l'image : comme si, là encore, une communion amoureuse secrète, un lien d'âme à âme avait donné naissance à cette vision. Un plan plus large montre le poète assis sur le rebord de sa propre fenêtre, regardant la mer audehors : les attitudes mêmes se rejoignent, chacun pensant à l'autre devant une fenêtre, dans la lumière. La cinéaste nous donne à voir l'impalpable, les liens que tisse l'amour par-delà l'espace et le temps et, au travers de ce moment

54. "on the viewless wings of Poesy» (J. Keats, "Ode to a Nightingale» [ Ode à un rossignol»], dans "Bright Star": The Complete Poems and Selected Letters, ouvr. cité, p. 237; je traduis). 
d'intense poésie né à la confluence des images, mots, voix, sons - ces quelques cordes très légères résonnant longuement dans le silence -, de cette communication spirituelle, ces correspondances profondes, saisit l'essence même du romantisme. L'onde circule entre ces deux rives, révélant tour à tour le visage de la jeune fille en plan rapproché, plein d'une joie intériorisée, les lignes de la lettre en surimpression, et le poète aperçu en plongée depuis la fenêtre à laquelle il se tenait plus tôt, silhouette sombre sur la grève face au miroitement de la mer immense, occupant presque tout le champ.

Sur cette voix off qui se poursuit, se fait jour une autre vision : Fanny, qui est partie se promener avec son frère et sa sœur en emmenant sa lettre d'amour, arrive dans la fascinante lande de bruyère, baignée de chants d'oiseaux, d'une lumière d'été très douce, de parfums presque perceptibles - la nature romantique toujours, alliée aux états d'âme. Comment ne pas penser ici à la quête mystico-amoureuse de la fleur bleue dans le roman Henrich von Ofterdingen de Novalis; ou bien alors à ces violettes parfois chantées par Keats, dont il disait à son ami Severn - qui en avait découvert, au cimetière protestant de Rome où le poète serait enterré peu après, de précoces floraisons, alliées à des pâquerettes - qu'elles étaient ses fleurs préférées... Toujours entièrement absorbée dans la lecture de sa lettre, la jeune fille en robe bleu sombre s'agenouille parmi le bleu-violet profond des fleurs; puis, à travers les ombres légères et floues de celles-ci au premier plan, se laisse tomber à la renverse, les bras étirés, auréolée de tout ce bleu, regardant vers le ciel, animée d'une joie ineffable, inconnue. Image de rêve d'une rare intensité, vision absolue du bonheur, de l'indivisible présence au monde. Les mots de la lettre de Keats sont à l'unisson de cet absolu : "Je souhaiterais presque que nous soyons des papillons et ne vivions que trois jours d'été - trois jours comme ceux-là avec vous je pourrais les remplir de plus de délices que cinquante années ordinaires n'en sauraient jamais contenir ${ }^{55}$." Le lien puissant établi par le poète entre le papillon, l'été, la brièveté et dans le même temps la densité d'être, n'est pas sans rappeler un passage du poème "To a Butterfly, 2" ("À un papillon, 2 ") de William Wordsworth, qui a pu l'inspirer : «Nous parlerons de soleil et de chants, / Et des jours d'été de nos jeunes années; / Doux jours d'enfance, qui étaient aussi longs / Que vingt jours le sont à présent ${ }^{56}$." Dans «To a

55. "I almost wish we were butterflies and liv'd but three summer days — three such days with you I could fill with more delight than fifty common years could ever contain." (J. Keats, Bright Star. Love Letters and Poems of John Keats to Fanny Brawne, introduction J. Campion, Londres, Penguin Books, 2009, p. 4; je traduis.)

56. "We'll talk of sunshine and of song, / And summer days when we were young; / Sweet childish days, that were as long/As twenty days are now." (W. Wordsworth, Poèmes, éd. bilingue, Paris, Gallimard, coll. «Poésie», 2009, p. I30; je traduis.) 
Butterfly, I» ("À un papillon, I»), son aîné évoque cette même volatilité du temps, incarnée de manière si poignante par cette créature ailée : "Reste près de moi - ne prends pas ton envol! / Reste un peu plus longtemps en vue! / [...] / Flotte près de moi; ne t'en vas pas encore! / En toi revivent les temps qui ne sont plus $[\ldots] /[\ldots] /$ Oh! heureux, heureux étaient les jours ${ }^{57}[\ldots]$ !» Dans le long poème narratif de Keats "Lamia», l'apostrophe implorante du jeune Lycius à la nymphe possède des accents semblables : "Car si tu disparaissais, je mourrais. / Reste! quoique Naïade des rivières, reste! / [...] / Par pitié, ne te fonds pas dans l'espace ${ }^{8}$ ! [...]» «Ici repose quelqu'un dont le nom était écrit dans l'eau" ("Here lies one whose name was writ' in water»), dit l'épitaphe gravée sur la tombe de Keats à Rome. Si tout chez lui se tient toujours à la lisière de la disparition, si la vie ne dure que le temps d'un battement d'ailes - et comment le poète qui devait mourir, si jeune, un an et demi à peine après cette première lettre, ne l'aurait-il pas pressenti, lui qui ailleurs écrivait : "Je sais le temps de mon être comptés9»-, ce qui donne un surcroît d'être, c'est ce qui donne un surcroît d'âme : l'amour - «mais alors le mystérieux, l'improbable, l'unique, le confondant et l'indubitable amour ${ }^{60} "$, dirait André Breton -, et la poésie, qui intensifient notre passage sur terre.

C'est dans ce plein jour du cœur et de la conscience que Fanny déclare à sa mère, pendant qu'autour d'elle volètent ou sont posés toutes sortes de papillons multicolores : «Mais quand je reçois une lettre [de John], je sais que notre monde est réel. C'est là que je veux vivre.» La jeune fille - c'est ainsi que la cinéaste l'imagine, dans la pureté et la radicalité de son amour - a en effet décidé de faire un petit élevage de papillons, dans sa chambre, "en notre honneur", précise-t-elle à Keats dans une lettre elle aussi imaginée ${ }^{6 \mathrm{r}}$, et tandis qu'elle écrit, dans un bocal près de l'encrier, un papillon bat doucement des ailes. Plus tard, un très gros plan s'attache à montrer dans toute sa splendeur un papillon bleu, posé sur un morceau d'orange. D'autres spécimens volètent dans des bocaux, ou à travers la pièce. Quelques magnifiques papillons aux ailes d'un bleu profond, irisé - comme en écho à celui du champ de fleurs, un peu plus tôt - sont posés certains sur la porte blanche de la chambre, d'autres sur

57. "Stay near me — do not take thy flight! / A little longer stay in sight! / [...] / Float near me; do not yet depart! / Dead times revive in thee [...] / [...] / Oh! pleasant, pleasant were the day [...]!» (Ibid., p. I20; je traduis.)

58. J. Keats, Poèmes et poésies, ouvr. cité, p. 212.

59. «Pourquoi ai-je ri ce soir?» ("Why did I laugh tonight?»), dans J. Keats, Seul dans la splendeur, éd. bilingue, trad. R. Davreu, Paris, Points, coll. «Poésie», 2009, p. 9I.

6o. A. Breton, Nadja, Paris, Gallimard, coll. «Folio», 200I, p. I59.

6r. Il ne reste aucune des lettres de Fanny Brawne à Keats, le poète les ayant détruites avant sa mort, sauf les toutes dernières, reçues en Italie, mais qu'il n'avait pas trouvé la force de lire et qui furent enterrées avec lui, sans avoir jamais été ouvertes. 
les mains et les bras de Fanny allongée sur son lit, ou de la petite Toots. Cette scène d'une mystérieuse beauté, développée, dans la continuité de celle parmi les fleurs, autour de la sublime et fragile présence des papillons - bientôt menacés par le chat, ou tristement retrouvés au terme de leur brève existence çà et là sur le plancher, au moment même où le désespoir gagnera Fanny -, occupe une place centrale, et ce n'est certes pas un hasard si Campion en fait un élément essentiel de sa poétique, en profonde affinité avec le sentiment keatsien du temps compté, de l'éphémère, du passage. Métaphore épistolaire s'incarnant dans le réel filmique, cet envol de papillons manifeste à son plus haut degré non seulement la capacité négative keatsienne et la romantisation du monde novalisienne, mais aussi «l'épanchement du songe dans la vie réelle ${ }^{62}$ » discerné par Nerval dans Aurélia. Comme si la poésie, des pages où elle avait été déposée deux siècles plus tôt, débordait dans la vie et s'y répandait, à la faveur d'un film, en images matérialisées, couleurs, textures, mouvements, en sons et silences.

\section{Rêves prémonitoires}

Cette osmose entre l'âme de Keats et la poésie manifestée à l'écran, cet "épanchement du songe dans la vie réelle» est particulièrement sensible dans une autre séquence où Campion, selon un procédé qui lui est cher, s'appuie à la fois sur l'œuvre, la correspondance et la vie du poète pour établir des liens subtils et cinématographiquement féconds entre elles. Elle nous montre, par une belle journée de printemps, Fanny et Keats qui ont enfin pu s'isoler quelques instants, sans chaperon - en l'occurrence Toots, restée avec ses camarades -, sous les ombrages d'un petit bois, pour leur premier tête-à-tête. Keats se met à raconter un rêve, fait la nuit précédente. Si la scène est imaginée par la cinéaste, les paroles qu'elle place dans la bouche du jeune homme sont, comme souvent, en partie empruntées à une lettre, avec quelques coupures et autres modifications. Il s'agit ici de la longue lettre-journal de février-mai I8I9 adressée à son frère George et sa belle-sœur Georgiana, où il évoque à la fois ledit rêve, inspiré par une lecture de L'Enfer de Dante, et le sonnet qu'il a composé par la suite, "A Dream, after reading Dante’s Episode of Paolo and Francesca» ("Un rêve, après avoir lu l'épisode de Paolo et Francesca de Dante»). Motion relate ${ }^{63}$ qu'en ce printemps de 1819, Keats avait rendu visite à Fanny, dans l'autre moitié de la maison de Wentworth Place qu'elle occupait désormais avec sa famille. Ils avaient alors lu ensemble le cinquième chant de L'Enfer, et plus

62. G. de Nerval, Les Filles du feu..., ouvr. cité, p. 296.

63. Voir A. Motion, Keats, ouvr. cité, p. 370. 
particulièrement le passage dans lequel Dante décrit l'amour qui naît entre Paolo et Francesca ${ }^{64}$ au cours de leur lecture partagée de l'histoire de Lancelot et Guenièvre... En retournant dans son aile de la maison, Keats était tombé dans un sommeil parcouru de rêves, dont naîtra le sonnet "Un rêve», et le passage suivant de la lettre à George et Georgiana - que la réalisatrice reprend, avec quelques modifications, dans une réplique de John :

J'avais passé maintes journées dans un état d'esprit plutôt déprimé, au cours desquelles j'ai rêvé que j'étais dans cette région de l'Enfer. Ce fut l'une des joies les plus délicieuses que $\mathrm{j}$ 'aie connues dans ma vie - Je flottais aux abords de l'atmosphère tourbillonnante qui y est décrite en compagnie d'une créature magnifique aux lèvres de laquelle mes lèvres étaient jointes depuis ce qui semblait une éternité $[\ldots]$ - même des cimes d'arbres fleuries se dressaient et nous nous reposions parfois sur elles avec la légèreté d'un nuage jusqu'à ce que le vent nous chassât de nouveau plus loin ${ }^{65}$.

Si les impressions du rêve, dans la lettre comme dans le film, sont empreintes de lumière, l'atmosphère du poème est pour sa part davantage sous l'influence directe de Dante, chargée de cette mélancolie dans le sillage des deux amants pris dans une damnation éternelle les vouant au second cercle de l'Enfer («Luxurieux, emportés par l'ouragan infernal $\left.{ }^{66} »\right)$. Chez Dante - comme si souvent chez Keats lui-même ${ }^{67}$-, c'est la perte qui domine, et amour et mort, éros et thanatos sont étroitement liés : «ainsi il m’en montra / et m’en désigna du doigt plus de mille / qu'amour ôta de notre vie ${ }^{68}$ ", "Amour nous a conduits à

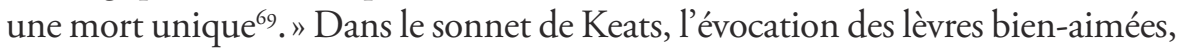
tout en restant fidèle à son aspiration apollinienne à la Beauté, est imprégnée de tristesse : «[...] Pâles étaient les douces lèvres que je vis, / Pâles étaient les lèvres que j'embrassai, et belle la forme / Avec laquelle je flottai, aux abords de cette mélancolique tempête ${ }^{70}$." Jane Campion établit un lien direct entre cette

64. Au XIII ${ }^{\mathrm{e}}$ siècle, Francesca de Rimini, épouse de Giovanni Malatesta, était tombée amoureuse de son beau-frère Paolo. Cette liaison leur sera fatale : Giovanni les tuera.

65. J. Keats, Lettres à Fanny et autres correspondances, ouvr. cité, p. 380-38I.

66. Dante, La Divine Comédie. L'Enfer, ouvr. cité.

67. "[...] et plus d'une fois / J'ai été presque amoureux de la Mort apaisante» : tout n'est-il pas dit dans ces quelques mots de l'"Ode à un rossignol "? Il est fréquent qu'une alternative finale entre l'amour et la mort soit suggérée. Voir à cet égard par exemple les poèmes «Bright Star», "To Fanny" ("À Fanny»), "This living hand... " "Cette main vivante... »), "I cry your mercy..." ("J'implore votre merci...»), ou maintes évocations dans les lettres à Fanny, comme celle-ci : "Je voudrais être dans vos bras plein de foi ou être frappé par la Foudre." (J. Keats, Lettres à Fanny et autres correspondances, ouvr. cité, p. 586.)

68. Dante, La Divine Comédie. L’Enfer, ouvr. cité.

69. Ibid., p. 65.

70. "[...] Pale were the sweet lips I saw, / Pale were the lips I kissed, and fair the form / I floated with, about that melancholy storm." (J. Keats, Bright Star. Love Letters and Poems of John Keats to Fanny Brawne, ouvr. cité, p. 87 ; je traduis). 
amoureuse évocation et la personne de Fanny - lien qui n'est pas explicite dans la lettre dont elle s'inspire -, puisqu' elle fait dire à la jeune fille, avec l'esquisse d'un sourire : «Quelles lèvres? Étaient-ce mes lèvres?» Question qui trouvera sa réponse, pleine d'émotion et de délicatesse, de sensuelle innocence, dans l'échange d'un premier baiser.

Ce dialogue fertile entre passages de Keats et événements du film, entre œuvre et vie, trouve un parfait accomplissement dans l'éclosion, un peu plus tard, d'une nouvelle vision, qui permet d'établir un lien très poétique avec d'une part ce sonnet inspiré par Dante et le rêve sous-jacent, d'autre part l' "Ode à un rossignol ». En effet, la caméra s'attarde en contre-plongée sur le fameux arbre où est censé se trouver le nid du rossignol, sur ses branches éblouissantes, symphonie florale baignée d'une lumière onirique, presque surexposée. Et lorsque la perspective se modifie et qu'une plongée vient révéler la cime de l'arbre, sur laquelle le poète amoureux est allongé face au ciel clair dans une inexprimable allégresse, c'est le rêve du poème, de la lettre, le rêve évoqué avant le baiser qui semble devenu réalité, s'incarnant jusqu'à recouvrir de sa lumineuse plénitude toute la surface de l'écran. Accomplissement romantique par excellence, tel que l'entrevoyait Novalis: "Le monde devient rêve, le rêve devient monde, / Et ce que l'on croyait advenu, / On peut le voir arriver au loin seulement ${ }^{71}$.» Et c'est comme si ce rêve-là, réinventé par le regard de Campion, avait dès lors été prémonitoire : comme s'il avait été porteur de ce baiser né sous nos yeux, de ces cimes d'arbre au-dessus desquelles flotterait ensuite, double des amoureuses silhouettes entrevues, la caméra «incarnante». Le chant du rossignol, par-dessus les cordes solairement égrenées, annonce l'ode à naître, et la très belle séquence où elle advient, sous l'arbre.

\section{Correspondances}

Certaines images-visions silencieuses contiennent ainsi en elles - et c'est là une manière fort subtile de rendre palpable la poésie de Keats, de la matérialiser au-delà des mots - des passages de poèmes ou de lettres. Tels ces plans successifs où, dans l'éclatante lumière des beaux jours, une abeille bourdonne sur la branche fleurie d'un arbre, une autre butine un coquelicot : presque une entrevision, de nouveau, mais qui, loin de traduire une attention purement esthétique à la nature, instaure véritablement un dialogue avec le poète. Dans

7I. "Die Welt wird Traum, der Traum wird Welt, / Und was man geglaubt, es sei geschehen, / Kann man von weitem erst kommen sehen." (Novalis, Heinrich von Ofterdingen, Francfort-surle-Main, Insel Verlag, coll. "Insel Taschenbuch», 1982, p. I55; je traduis et souligne.) 
la rencontre en très gros plan de cette fleur et de cette abeille, comment ne pas reconnaitre en effet l'écho de maints poèmes de Keats : «Et comme les fleurs nouvelles au bruissement matinal des abeilles ${ }^{72}$ " («Lamia»), "Avant aussi que les abeilles / Bourdonnent autour des trètles globulaires et des pois de senteur ${ }^{73}$ ", " ô toi, à qui / Les figuiers aux larges feuilles ont dès maintenant prédestiné / Leur récolte mûrissante; les abeilles ceinturées d'or / Leur miel blond; les prés de nos campagnes / Les plus somptueuses fleurs de fèves et les coquelicots des blés ${ }^{74}$ » («Endymion»), «Mais la rose se laisse croître sur la lande / Pour s'offrir aux baisers du vent et nourrir l'abeille qui rend grâce ${ }^{75}$ » ( La Gloire, II»), "à faire éclore davantage, / et encore davantage, de fleurs tardives pour les abeilles ${ }^{6}{ } \ldots$ Ou encore, étonnamment proche du plan de Campion, cet hommage du peintre Haydon à l'extrême sensibilité de son ami Keats : «Le bourdonnement de l'abeille, la vue d'une fleur, le scintillement du soleil semblaient faire vibrer sa nature ${ }^{77}$ !» Mais c'est, aussi et surtout, à un passage de sa lettre du I9 février I8I8 à John Hamilton Reynolds que l'on songe, caractéristique de son esthétique nuancée, attentive à la part dite féminine de l'être, et de sa philosophie empreinte de capacité négative :

C'est une vieille comparaison destinée à nous exhorter que celle de la ruche : il me semble toutefois que nous devrions plutôt être la fleur que l'Abeille; car c'est une idée fausse, qu'on gagne plus à recevoir qu’à donner. Non, celui qui reçoit et celui qui donne sont égaux au regard des bienfaits. [...] n'allons donc pas nous affairer à butiner comme une abeille, bourdonnant çà et là avec l'impatience de qui sait ce qu'il doit obtenir : ouvrons nos pétales comme une fleur, soyons passifs et réceptifs ${ }^{78}[\ldots]$

En montrant, dans le plan suivant, Fanny et Keats qui, accompagnés de la petite Toots, se promènent dans le jardin en pleine floraison, sous l'œil attentif de la famille qui les observe depuis la fenêtre de la cuisine - «ils cherchent les fleurs les plus odorantes dans le jardin ", justifie Sam -, et en faisant de surcroît malicieusement commenter à la servante : «Monsieur Keats fait l'abeille», Jane Campion file la métaphore de la lettre, suggérant clairement le lien amoureux entre les deux jeunes gens.

72. J. Keats, Poèmes et poésies, ouvr. cité, p. 207.

73. Ibid., p. 63.

74. Ibid., p. 69.

75. J. Keats, Seul dans la splendeur, ouvr. cité, p. I05.

76. "[...] to set budding more, / and still more, later flowers for the bees [...]" (J. Keats, "Bright Star": The Complete Poems and Selected Letters, ouvr. cité, p. 249; je traduis).

77. Cité par R. Davreu, dans J. Keats, Seul dans la splendeur, ouvr. cité, p. 7.

78. J. Keats, Lettres à Fanny et autres correspondances, ouvr. cité, p. I28-I29. 
La cinéaste utilise de manière créative d'autres passages empruntés à des lettres, qui viennent nourrir les répliques de Keats, dans des contextes différents de ceux d'origine. La langue épistolaire keatsienne devient par là même souvent langue d'échange filmique du personnage Keats. Ainsi, les phrases que prononce le poète au cours de la leçon de poésie donnée à Fanny sont issues, avec quelques modifications, de lettres à différents correspondants : le développement sur l'art de composer provient de la lettre du 27 février I8I8 à John Taylor : " et cela me conduit à un autre axiome. Que si la Poésie ne vient pas aussi naturellement que les Feuilles sur un arbre il vaudrait mieux qu'elle ne vienne pas du tout ${ }^{79}{ }^{\text {». }}$ Quant aux considérations sur ce que Keats appelle le "Caractère poétique», elles trouvent leur source dans une lettre du 27 octobre I8I8 à Richard Woodhouse, célèbre pour la notion de "poète caméléon" qui y est développée :

Un poète est la chose la moins poétique qui soit; car il n’a pas d'identité - il est constamment forme - et matière d'un autre Corps - Le Soleil, la Lune, la Mer, les Hommes et les Femmes, créatures impulsives, sont poétiques et possèdent en eux un attribut permanent - le poète n'en possède aucun; il n'a aucune identité - il est certainement la moins poétique des créatures de Dieu ${ }^{80}$.

Autre exemple de réutilisation de la correspondance, lorsque John se fâche contre Brown, qui a fait une mauvaise plaisanterie en envoyant un message de Saint Valentin à Fanny, sa réplique "Les affections du cœur sont sacrées, l'ignorez-vous?» est tirée d'une lettre à Benjamin Bailey du 22 novembre I8I7 : "Il n'est rien dont je sois certain sinon de la sainteté des affections du cœur et de la vérité de l'Imagination ${ }^{8 \mathrm{I}}$."

Les lettres en tant que telles figurent en bonne place dans le film, à commencer par celles, bouleversantes, à Fanny, qui ponctuent Bright Star de leur puissant lyrisme en voix off, voix intérieure du poète, ou dont, plus rarement, leur destinataire lit un passage à voix haute. Pour l'une d'entre elles, sa voix se superpose un instant, avec émotion et fort symboliquement, à la voix off de Keats - délicate surimpression sonore par laquelle se crée un dialogue amoureux transgressant les limites spatio-temporelles -, sur la fin de la phrase, qu'elle répète : «Mais si vous m’aimez pleinement, nous braverons le feu de la passion nimbés de la rosée des plaisirs.» Cependant d'autres fragments de sa correspondance sont intégrés au déroulement des événements : les poignantes dernières lettres envoyées à l'automne I820, depuis l'Italie, à Brown ainsi qu’à Mrs Brawne. Keats n'avait plus la force d'écrire à Fanny ni de la lire, mais elle ne quittait pas ses pensées. Les lettres sur lesquelles il est encore donné à la

79. Ibid., p. I34.

8o. Ibid., p. 263-264.

8I. Ibid., p. 86. 
jeune fille de poser le regard - ce regard dans lequel toujours passe une sourde mélancolie, une attente anxieuse informulée - et dont elle reconnaît si bien l'écriture, sur l'enveloppe, ne lui sont désormais plus destinées : celles que Brown tient entre les mains au retour de sa longue absence, ou celle, adressée à Mrs Brawne, dont un très gros plan la révélant posée contre la cafetière restitue la muette puissance d'émotion. Il est frappant de noter le parfait naturel avec lequel, ici comme ailleurs, des passages - plus ou moins librement repris de Keats - font leur apparition dans le fil des événements. Un naturel caractéristique de l'approche de Campion, qui intègre le texte keatsien avec fluidité et évidence aux dialogues, à la narration, le laissant suivre son cours comme une profonde nécessité intérieure, un fleuve souterrain, jusqu'à en irriguer la vie même des personnages. Lorsque Mrs Brawne rapporte, en souriant : «Il dit que c'est comme un rêve", son récit est teinté de sa propre perspective positive - ou se voulant rassurante envers Fanny - de la situation. Celle-ci est pourtant désespérée, comme en témoigne le passage exact de Keats, dans sa lettre du 24 octobre I820: «si je me portais bien il y aurait dans ce port de Naples de quoi remplir une main de papier - mais l'on dirait un rêve - tout homme qui peut ramer et marcher et parler me semble un être différent de moi - je ne me sens pas de ce monde ${ }^{82} »$. Lorsque Brown exprime amèrement ses remords d'avoir abandonné Keats, les mots qu'il emploie sont, cette fois, exactement ceux d'une de ses lettres écrites après la mort du poète : «Quelle qu'ait été la profondeur de mon affection pour lui, j’ignorais jusque-là l'intensité de son emprise sur mon coeur ${ }^{83}$."

La déchirante séquence des adieux nocturnes des amoureux, où Fanny ne peut retenir ses larmes et sa révolte face à cette vie absurde qui va irrémédiablement les séparer, est quant à elle née d'un passage d'une lettre de Keats à Brown du 30 septembre I820, et où les paroles rapportées de la jeune fille sont presque identiques à celles qu'elle prononce dans cette scène :

Quelques-unes des expressions qu'elle avait coutume d'utiliser au cours des soins que j'ai reçus en dernier à Wentworth Place résonnent à mes oreilles $-Y a-t-i l$ une autre Vie? Vais-je me réveiller pour découvrir que tout ceci neétait qu'un rêve? Il le faut nous ne pouvons avoir été créés pour pareille souffrance ${ }^{84}$.

82. Ibid., p. 602-603.

83. "Much as I have loved him, I never knew how closely he was wound about my heart." (Cité dans A. Motion, Keats, ouvr. cité, p. 53I; je traduis et souligne.)

84. J. Keats, Lettres à Fanny et autres correspondances, ouvr. cité, p. 6oI; je souligne. 


\section{Flamboiements}

Chaque fois que Jane Campion introduit un élément de la biographie ou de l'œuvre du poète, c'est comme si elle lui permettait de vivre une vie autonome, le laissait déployer ses ailes et entraîner immanquablement un lyrisme nouveau dans son sillage, venant prolonger et amplifier celui de Keats. Dans les creux, les intervalles, les points de suspension mêmes de cette vie connue et inconnaissable s'insinue et s'invente la poésie. Infinie puissance incantatoire des ellipses, à laquelle est sensible tout cinéaste véritable : en laisser parfois simplement vibrer le vide lumineux, en recueillir, intactes, les plus infimes résonances; et parfois, l'habiter avec grâce, y dessiner des paysages intérieurs, accordés à l'âme fervente des amants : des visions. Quelques lignes d'Andrew Motion suffisent à donner naissance à des scènes d'une grande intensité. Ainsi, au sujet des quelques semaines de l'été I 820 où, parenthèse de paix avant son départ vers l'Italie et les adieux définitifs, Keats très malade est accueilli par Mrs Brawne et entouré de soins et d'affection par toute la famille, le biographe commente : «Keats se sentit plus en sécurité et chez lui qu'il ne l'avait été depuis des mois, et plus heureux, devait-il dire plus tard, qu'il ne l'avait été de toute sa vie $e^{85}$.» Campion filme ces instants de grâce avec une tendresse teintée de mélancolie et une nostalgie radieuse, nimbée de cette lumière de fin d'été si sensible dans l'ode «To Autumn» ("À l'automne») de Keats : «Saison de brumes et de moelleuse abondance! / Amie intime du soleil qui mûrit [...]. / [...] / Où sont les chansons du Printemps? Oui, où sont-elles? / N'y pense plus, tu as toi aussi ta musique ${ }^{86}$.» Ce sont ces heures douces de rémission de la maladie, de forces qui reviennent un peu, autour d'une assiette de soupe et parmi l'amour des siens - ces quelques regards, sourires et gestes affectueux unissant le poète, sa fiancée et la petite Toots aux joues empourprées comme si elle avait mangé des boutons de rose, plaisante Keats, et suspendant provisoirement le temps compté - «Jusqu'à ce qu'elles croient que les chaudes journées ne cesseront jamais ${ }^{87}$ »... C'est, one-two-three, one-two-three, cette émouvante petite valse improvisée en plein champ, dans le balancement léger d'un châle rouge et d'un chapeau de paille suspendus à une branche, le joyeux désordre des tables et des chaises sous les frondaisons, les éclats de rire, les mains qui frappent le rythme tandis que

85. "[...] Keats felt more safely at home than he had done for months, and happier, he said later, than he had ever been in his whole life." (A. Motion, Keats, ouvr. cité, p. 527; je traduis).

86. "Season of mists and mellow fruitfulness! / Close bosom-friend of the maturing sun [...]. I Where are the songs of Spring? Ay, where are they? I Think not of them, thou hast thy music too [...]" (J. Keats, "Bright Star": The Complete Poems and Selected Letters, ouvr. cité, p. 249-250; je traduis). 87. "Until they think warm days will never cease» (ibid., p. 249; je traduis). 
virevoltent la mère et le fils, les regards radieux, les fleurs dans les cheveux de John, la brise qui caresse les amoureux, le trio enjoué de Fanny, Keats et Toots, les premiers pas tendrement esquissés d'une danse avec le bien-aimé. Vision de bonheur, saturant l'image de plénitude, dans la complicité d'une saison dorée. Entre-deux intense et fragile comme un rêve, teinté d'absolu et d'éphémère. «Et ne reviens pas! Il n'y a pas d'automne par ici», dit solennellement la petite Toots à une feuille morte qu'elle va déposer à l'écart dans l'herbe, ses cheveux roux flamboyant dans les incandescences ultimes de l'été. Poésie d'enfance, petit rituel magique pour chasser le malheur, le tenir à distance. Mais déjà la menace refait surface : John tousse, chancelle - la danse reste inachevée.

Évoquant la quiétude provisoire de ce dernier mois d'août, Motion note : «Il passait de longues heures à somnoler ou à regarder par la fenêtre, ou à parler avec Fanny de la vie quils ne partageraient jamais ${ }^{88}$." De cette simple fin de phrase, Campion tire une séquence bouleversante de mélancolique poésie, de passion contenue, d'espoir désespéré, puisqu'il faut pour s'empêcher de sombrer jeter ses dernières forces, avant l'adieu, dans l'imagination de ce qui ne sera jamais. Au dernier soir, dans le clair-obscur des bougies, la lumière mordorée à l'unisson de cette flamme inextinguible qui danse en eux, enlacés sur le lit, Fanny et Keats s'obstinent à inventer l'avenir avec une infinie tendresse - à romantiser le monde :

Keats : - Faisons comme si je revenais au printemps.

Fanny : - Vous reviendrez, je le sais.

Keats : - Nous vivrons à la campagne.

Fanny :- Près de maman.

Keats : - Notre chambre donnera sur un petit verger, avec à l'horizon une montagne embrumée.

Fanny : - Nous cultiverons un jardin où pousseront toutes sortes de fleurs sauvages.

Keats : - Nous nous coucherons quand le soleil sera encore haut...

Fanny : - Et quand il fera nuit, la lune brillera à travers les persiennes.

Keats : - Je vous tiendrai serrée contre moi...

Tandis qu'avec une chaste volupté ils laissent émerger en eux, et entre eux, cette vision intérieure d'un amour accompli, un gros plan - la caméra ne s'est jamais aventurée aussi près de leurs âmes, mais avec délicatesse, comme au seuil d'un temple - saisit celle-ci dans son évanescence même, au détour de ces larmes brillant dans leurs yeux, à la lumière des bougies, cette infime caresse sur un

88. "He spent long hours dozing or staring out the window, or talking with Fanny about the life they would never share.» (Ibid., p. 532 ; je traduis et souligne.) 
visage, cette main serrée un instant, ces fugitifs baisers sur une silhouette, une étoffe moirée, gestes-promesses d'un avenir entrevu qui n'aura pas lieu. "Le toucher a une mémoire", dit John à Fanny. "Je sais", répond-elle. La phrase qu'il prononce fait partie d'un poème écrit pour elle ("Lines to Fanny», "Vers pour Fanny») : «Le toucher a une mémoire. Ô dis-moi, mon amour, dis-moi, / Que puis-je faire pour l'abolir et retrouver / Mon ancienne libertét ${ }^{89}$ ?»

\section{Visions entrelacées}

Les poèmes en tant que tels, comme on a pu le voir pour l'" Ode à un rossignol», apparaissent au fil de Bright Star, lus ou récités, en voix off/ intérieure ou en voix in, toujours dans un rapport d'évidence et de spontanéité avec tout ce qui les entoure et les voit naître. Les premiers vers entendus sont ceux du début d' "Endymion": lus à haute voix d'abord par Toots, qui est allée chercher avec Sam le recueil pour leur sœur - et dès les premiers mots, quelque chose se passe en Fanny, quelque chose a irrémédiablement eu lieu, elle suspend son ouvrage -, puis par la jeune fille elle-même, qui, diaphane dans cette lumière imprégnant de sa transparence jusqu'aux pages doucement feuilletées, penchée sur ce livre à la couverture bleu pâle un instant caressée, s'avance vers des territoires inconnus, vers ce cœur du mystère : rencontre déjà amoureuse, par poésie interposée, avec l'âme d'un être qu'on tiendrait là, entre ses mains : "Une chose de beauté est une joie pour toujours : / Son charme s’accroît; jamais elle / Ne glissera dans le néant ${ }^{9 \circ}[\ldots] »$.

Fanny reprendra plus tard ce même début d'«Endymion» en présence de son auteur, lors du bal - premiers instants de complicité. Et, tandis que son intérêt pour la poésie croît parallèlement à ses sentiments pour le jeune poète, ce sont des vers de «La Veille de Sainte-Agnès " - ballade si inspirante pour Jane Campion - qu'elle récitera à un Brown quelque peu railleur à l'égard de son soudain engouement : "Comme elle se hâtait, le flambeau s'éteignit; / Sa petite fumée, clair de lune évanescent, mourut : / Elle ferma la porte, palpitante, en alliance intime / Avec les esprits de l'air et les vastes visions ${ }^{91}[\ldots]$ [.. C’est ce même Brown qui fera part à Keats de toute la profondeur de son émotion et de son admiration après avoir récité un autre passage de ce poème : «Bientôt,

89. "Touch has a memory. O say, love, say, /What can I do to kill it and be free / In my old liberty?" (J. Keats, "Bright Star": The Complete Poems and Selected Letters, ouvr. cité, p. 36I; je traduis.) 90. "A thing of beauty is a joy for ever: / Its loveliness increases; it will never / Pass into nothingness [...]» (ibid., p. 63; je traduis).

91. J. Keats, Poèmes et poésies, ouvr. cité, p. I63. 
tremblante dans son nid moelleux et glacé, / En une sorte d'évanouissement éveillé, inquiète elle s'étend ${ }^{92}[\ldots] »$.

À son tour, Fanny exprimera à celui qu'elle aime toute sa foi en sa poésie et, malgré ce terme d' "impossibilités » qu’il a employé dans une lettre et qui la tourmente - «Je ne puis dire oubliez-moi - mais il me faut faire remarquer certaines impossibilités en ce monde ${ }^{93}$ "-, en leur amour et leur avenir commun : "Je ne peux vous quitter. Votre nouveau recueil de poèmes ${ }^{94}$ est si prometteur. John, ils sont plus beaux que tout ce que j'ai jamais lu de Mr Coleridge, de Mr Wordsworth, même de Lord Byron.» C'est là le point de départ de l'une des plus belles séquences du film : une sorte de duo amoureux porté par la poésie, ou de duo poétique porté par l'amour, une longue récitation à deux voix de l'ensorcelante ballade de «La Belle Dame sans merci» - six des douze strophes que compte le poème -, un dialogue en apesanteur où s'entrelacent poésie verbale et cinématographique, amour de légende et amour vécu, présent et intemporel, réel et art. Fanny, les yeux posés sur John, emplis d'une mélancolie douce accordée à l'atmosphère du poème, en dit la première strophe : «Ah! qui peut te faire souffrir, chevalier en armes / Errant pâle et solitaire! / Les joncs sont desséchés au bord du lac, / Aucun oiseau n'y chantes5." Le contrechamp dévoile un Keats ému, qui poursuit - en passant directement, et ce n'est certes pas anodin, à la quatrième strophe, qui voit apparaître, tel un double évanescent de Fanny, le personnage de la Belle Dame - : «J'ai rencontré une dame dans les prés, / D'une grande beauté - la fille d'une fée; - / Ses cheveux étaient longs, ses pieds légers / Et ses yeux sauvages. » Son regard, attaché à Fanny, est chargé d'une sourde intensité tandis qu'il prononce cette strophe, puis la suivante, qui est la sixième du poème : "Je l'assis sur mon coursier paisible / Et ne vis rien d'autre tout le long du jour; / Car elle se penchait de côté et chantait / Une chanson de fée. " L'émotion, bien que contenue, intériorisée, est palpable tout au long de cette séquence, nourrie par tout le jeu subtil des regards, de micro-mouvements, par la vibration des voix, la modulation du souffle, la circulation de la poésie entre Keats et Fanny comme une vague, une

92. Ibid., p. 165 .

93. "I cannot say forget me-but I would mention that there are impossibilities in the world." (J. Keats, Bright Star. Love Letters and Poems of John Keats to Fanny Brawne, ouvr. cité, p. 30 ; je traduis.) Cette occurrence relève de nouveau du procédé, cher à Campion, d'entremêlement de l'œuvre et de la vie des personnages à l'écran.

94. Il s'agit du recueil Lamia, Isabella, The Eve of St Agnes and Other Poems, paru le I ${ }^{\mathrm{er}}$ juillet I820, qui sera favorablement accueilli par la critique, et dont le poète dédicace des exemplaires aux Brawne à la fin du film.

95. Le poème est cité pour partie dans la traduction de P. Gallimard (J. Keats, Poèmes et poésies, ouvr. cité, p. I79-I80, pour ce passage et les deux suivants), les autres citations (signalées en note) étant issues d'une traduction personnelle. (Voir infra, notes 96 et 98.) 
onde, un courant sous-marin qui nous parle d'eux, et par lequel ils se parlent à demi-mot. Fanny continue, avec une gravité tendre : "Elle trouva pour moi des racines d'un goût exquis, / Du miel sauvage, et la manne de la rosée; / Et sûrement dans une langue étrange elle dit : / Je t'aime véritablement ${ }^{96}$." Sur l'avant-dernier vers, John a fermé les yeux, et l'on sent des larmes y monter; ils sont baissés sur les mots d'amour prononcés par Fanny-la Belle Dame, au dernier vers : comme si toujours il s'agissait d'effleurer, d'osciller entre le révélé et le caché. Beckett disait de Keats : "Il est de tous celui que je préfère, parce qu'il ne tape pas du poing sur la table. J'aime cette terrible douceur ${ }^{97}$ ", et l'art de Campion est en harmonie avec cette délicatesse passionnée. Une série de champs-contrechamps s'attache avec pudeur à la beauté recueillie de ces visages, s'écoutant, se répondant et ressentant au plus profond de soi les mots de l'autre. Mots d'amour, dans la "langue étrange» et "véritable» du poème, dont seul un cinéma à l'écoute des dimensions secrètes de l'être, attentif aux plus infimes variations du visible et de l'invisible, un cinéma allié des métaphores tel que celui qui habite Bright Star, est capable de restituer, intact par-delà les siècles, le souffle tellurique. Dans l'entrecroisement de ces regards, de ces mots, de ces silences, c'est le cinéma et la poésie mêmes qui entrelacent leurs forces de surgissement, d'éblouissement.

Chacun enchaîne à présent, dans une intensification sensible de l'émotion, sur le vers prononcé par l'autre :

Keats : «Elle m'entraîna dans sa grotte d'elfe»

Fanny : «Et là elle versa des larmes et poussa des soupirs pleins de douleur»

Keats : «Et là je fermai ses yeux sauvages, sauvages / De quatre baisers. / Et là, me berçant, elle m'endormit / Et là je rêvai - Ah! malheur à moi! - / Le dernier rêve que j'aie jamais rêvé / Sur le flanc de la froide colline ${ }^{98}$.»

Ces deux derniers vers récités par le poète prennent ici un relief extraordinaire : comment ne pas penser, dans cette évocation de l'ultime rêve, où son regard se pose sur la jeune fille, du tout dernier rêve fait sur une terre froide, à cette mort qui s'approche, mal contenue par l'amour, pourtant immense, qui l'unit à Fanny, digue paradoxale puisqu'aussi bien, de par son inaccomplissement, son inachèvement, instrument involontaire de cette mort, comme dans « La Belle Dame sans merci». Ce déchirant paradoxe est clairement établi dans ses dernières lettres : «Mon cher Brown, que faut-il que je fasse? Où puis-je

96. Voir note précédente. Je traduis ce passage du poème.

97. "I like him the best of them all, because he doesn't beat his fists on the table. I like that awful sweetness [...]" (cité par A. Motion, Keats, ouvr. cité, p. 577; je traduis).

98. Je traduis ce passage du poème. 
chercher une consolation ou du repos? Si j'avais une chance de guérir, cette passion me tuerait 99 .» Il écrit encore :

Ce pour quoi je désire le plus vivre sera la véritable cause de ma mort. Je n'y peux rien. Qui y peut quelque chose? Serais-je en bonne santé que cela me rendrait malade, alors comment le supporter dans cet état? [...] J'appelle nuit et jour la mort qui me délivrerait de ces souffrances, et puis je souhaite que la mort s'éloigne, car la mort détruirait même ces souffrances qui valent mieux que rien ${ }^{\text {roo }}$.

\section{«Étoiles innommées ${ }^{\mathrm{IOI}}$ »}

C'est aussi dans une forme de dialogue, mais différé, par-delà la mort, qu'apparaît le poème «Bright Star». Par-delà la mort, et dans le même temps profondément inscrit dans la vie, porteur de l'image d'amour la plus dense, la plus sereine qui soit. Comme nombre de poèmes de Keats, comme cette double vision qui ouvre et clôt pratiquement le film - entre le blanc-fête des commencements, de tous les possibles, et le noir ultime du deuil -, le sonnet et la manière dont il se manifeste sont caractérisés par une certaine ambivalence. C'est dans un contexte de pure harmonie amoureuse et de douceur de vivre qu'il est entendu pour la première fois : Fanny et Keats, enfin seuls, partagent quelques instants de profonde tendresse et complicité. Le poète, la tête appuyée contre l'épaule de sa bien-aimée, leurs mains entrelacées, se met à lui réciter le passage final : «Reposant sur le sein mûri de mon bel amour, / Pour sentir à jamais le doux souffle qui l'anime, / Éveillé à jamais dans une délicieuse agitation, / Pour toujours, toujours entendre sa tendre respiration ${ }^{\mathrm{102}}[\ldots]$ ». À la jeune fille qui lui demande quel est ce nouveau poème, il répond : «Le vôtre». C'est en effet sous le signe de Fanny, de cette «Brillante étoile» qu'elle représente à ses yeux, qu'est placé ce sonnet ${ }^{103}$, à l'instar d'autres œuvres qui lui sont dédiées. Keats dit alors le début de ce poème d'amour : «Brillante étoile! Que ne suis-je comme toi immuable, / Non seul dans la splendeur tout en haut de la nuit ${ }^{104}{ } \ldots .$.

99. J. Keats, Lettres à Fanny et autres correspondances, ouvr. cité, p. 605.

Ioo. Ibid., p. 6oI.

IOI. "Ode à Psyché», dans J. Keats, Seul dans la splendeur, ouvr. cité, p. IOI.

IO2. Je traduis ce passage du sonnet.

I03. Si la datation précise de ce poème est difficile, Motion penche plutôt pour octobre I8I9 (voir A. Motion, Keats, ouvr. cité, p. 472). Fanny Brawne le recopiera sur l'exemplaire de L'Enfer de Dante que Keats lui avait donné. Ce dernier le retranscrira, à bord de la Maria Crowther qui l'emmène en Italie, pour son ami Severn qui l'accompagne, sur la page de garde d'un recueil d'œuvres de Shakespeare.

I04. Je reprends ici et dans la suite du développement (sauf pour le passage final déjà cité, voir supra, note IO2, et infra, note I07) la traduction de Fouad El-Etr, utilisée dans le film. 
«Bright Star» s'élèvera encore une fois à la toute fin du film, mais dans une obscurité désolée, une douloureuse solitude contrastant avec la lumière de cette scène heureuse. Dans la pâleur naissante d'un matin d'hiver, revêtue des habits du deuil qu'elle a cousus toute la nuit, Fanny s'échappe de la maison, en direction du bois et des sentiers autrefois parcourus avec son bien-aimé - et lorsqu'elle passe entre les battants de la porte d'entrée, silhouette sombre se découpant sur fond de neige et de brume au loin, c'est une image claire-obscure des Trois Lumières de Fritz Lang - Der müde Tod (I92I), littéralement «la Mort lasse» selon le titre original - qui affleure à la mémoire : celle où la jeune femme franchit la haute muraille de la Mort, passant par cette "porte étroite» en forme d'ogive gothique qui s'y ouvre entre les mondes afin de retrouver, de l'autre côté, son fiancé que la Mort lui a enlevé. Orphée féminin sur les traces de son amour perdu, Fanny s'avance seule - à peine suivie à distance par son frère Sam, présence floue, plus discrète que jamais - à travers l'opacité du bois, récitant, comme une incantation, tout le poème, dont le premier vers s'est esquissé en voix off sur un plan général du paysage. Marche lente, lancinante dans la lumière bleutée de l'aube, épousée en un long travelling latéral qui place la jeune femme presque constamment dans la moitié droite du cadre, en avant de l'image, comme si elle entraînait toute la nature dans son sillage, ces infinies silhouettes d'arbres en fond de champ. Une nature profondément romantique, comme toute la scène : toujours accordée aux états d'âme - de l'endeuillée, mais aussi du poème qui résonne dans l'air glacé. Par l'amour, la poésie et un cinéma capable de saisir leur essence, une singulière osmose s'établit entre l'âme de Fanny, celle de Keats, et celle de la nature. Une nature-miroir, à l'instar du visage de Fanny, extrêmement mais sobrement expressif, pâle, enveloppé des échos clairs de son col, de la neige, de la lumière hivernale, contrastant avec les tonalités sombres de sa chevelure, son chapeau et ses vêtements. Visage-univers, sur lequel naissent et passent tant d'émotions sourdes, tant d'indicible, tant d'abîmes, où dans le profond silence du matin, à peine troublé de quelques appels d'oiseaux çà et là - mais «Où sont les chansons du Printemps? Oui, où sont-elles?»-, le moindre frémissement de la poésie de Keats trouve un écho et un refuge, traçant un chemin secret de lueurs et de larmes dans ses yeux, sur ses joues.

Un léger vent joue avec le ruban noir de son chapeau. Fanny semble vivre, et revivre, chaque mot du poème, d'une profonde vie intérieure qui transparaît simplement au grand jour : "Non seul dans la splendeur tout en haut de la nuit»... et elle cherche du regard, au fond du ciel d'hiver, l'invisible étoile. «Être heureux avec vous paraît tellement impossible! Il faudrait une étoile plus heureuse que la mienne! cela ne sera jamais ${ }^{105}$ ", écrivait Keats dans sa

I05. J. Keats, Lettres à Fanny et autres correspondances, ouvr. cité, p. 587. 
dernière lettre à Fanny - et dans sa dernière lettre à Brown : «C'était là mon étoile prédominante ${ }^{106}$ !» Étoile double, tout à la fois signe d'amour et signe de mort, à laquelle il faut la nuit pour exister. La jeune femme poursuit, presque en somnambule : «De la nature patient ermite sans sommeil» : et ce mot - «sleepless»-, légèrement accentué, devient comme un reflet d'elle-même, de sa nuit blanche de deuil, tandis que, sur "Les eaux mouvantes dans leur tâche rituelle", une souffrance plus grande encore envahit son visage, comme une crue, une marée montante de larmes difficilement contenue, un désespoir dont le mouvement suivrait la courbe fluide du sonnet. Sa manière d'habiter intensément tous ces vers, toutes ces visions, crée des liens puissants entre l'éternité du poème et ce douloureux présent qu'il scande, qu'il remodèle. "Non-mais toujours immuable, toujours inchangé» : la négation prend une dimension nouvelle dans sa bouche, devenant déchirante exclamation, comme en écho à son refus de cette mort, de cette absence, et une aspiration à retrouver cet état d'immuabilité, cette permanence évoqués dans le vers. Et lorsque, un tremblement dans la voix, elle reprend le passage final du poème ${ }^{\mathrm{ro7}}$, entendu pour la première fois en des jours heureux : «Reposant sur le sein mûri de mon bel amour...", et que, parvenant à "Pour sentir à jamais» - «To feel for ever»-, elle prononce ces mots avec ferveur, les yeux clos et pleins de larmes, nous savons ce qu'elle ressent et revit à jamais, pour l'avoir vécu avec elle, dans la lumière des beaux jours révolus et l'amoureuse présence du poète, lorsque cette «Brillante étoile» avait point pour la première fois à l'écran. Ce «doux souffle» dont Keats voulait se mettre éternellement à l'écoute, voilà qu'il se perpétue sans lui - sans sa propre «tendre respiration", et la jeune femme, submergée par la douleur, n'arrive presque plus à poursuivre : l'on se souvient alors de cette même difficulté à respirer du poète, tandis qu'avec Fanny il récitait «La Belle Dame sans merci»... «Éveillé à jamais» - le visage baigné de larmes, la fiancée endeuillée marche droit devant elle, le regard perdu au loin, dans l'inaccessible, la mort en face. Marche lente d'amour et d'absence qui dure le temps suspendu d'un poème, le temps hors du temps d'un long travelling élégiaque. Une douceur désespérée dans la voix, Fanny atteint le dernier vers de «Bright Star» - qui est aussi le dernier vers du film -, que John, la première fois, n’avait pas prononcé : «Et vivre ainsi à jamais - ou bien défaillir dans la mort.» La mort, qui est le dernier mot - qui a le dernier mot, juste avant un fondu au noir scellant la disparition et la nuit. Terme provisoire d'une marche sans fin, où tout l'arrière-plan de la forêt, du « réel» reste flou, et Fanny seule dans l'acuité du poème, des sentiments et des pensées, comme si le regard

Io6. Ibid., p. 607.

I07. Je traduis ce passage qui clôt le poème. 
était entièrement tourné vers son monde intérieur, absorbé par lui, à l'instar de ce que vit l'errante elle-même, en ces instants où elle s'avance comme morte à ce monde où Keats ne vit plus. Elle semble évoluer à son tour au sein de ce "rêve" dont parlait le poète dans sa lettre de Naples à Mrs Brawne, et les mots de sa dernière lettre à Brown viennent hanter la mémoire : "J'ai un sentiment permanent que ma vie réelle est passée, et que je mène une existence posthume ${ }^{108}$." Plus que jamais, dans ces ultimes images, ces ultimes mots, amour et mort apparaissent liés : plus que jamais, l'âme de Keats y vibre, et l'âme de Fanny s'y surimpressionne. Un long plan noir s'inscrit dans le sillage de cet effacement, puis, comme deux étoiles l'une après l'autre surgies sur ce fond de nuit, deux cartons en caractères blancs se succèdent en silence, avec une poésie de cinéma muet. Le premier, venant prolonger l'errance, rappelle : "Fanny Brawne arpenta la lande des années durant, souvent jusque tard dans la nuit. Jamais elle n'oublia John Keats ni n'ôta sa bague.» Le second rend hommage au poète : «Keats mourut à vingt-cinq ans, persuadé d'avoir échoué. Il est aujourd'hui reconnu comme l'un des plus grands poètes romantiques. » Keats et Fanny se trouvent ainsi reliés par-delà cette nuit.

Comme si la voix de John répondait à présent à Fanny, elle récite off sur le fond noir du générique l'"Ode à un rossignol ", elle aussi dans son intégralité, elle aussi poème des temps heureux, accompagnée par la sérénade-leitmotiv de Mozart. La voix poétique de Keats, sa voix de poésie - que ce soit à travers le "Bright Star» récité par Fanny, ou cette "Ode à un rossignol » portée par sa propre voix «humaine» - s'élève comme une voix toujours vivante, qui nous parlerait simplement depuis un autre espace-temps, se déployant dans, et malgré toute nuit. «Je n'ai jamais bien su tirer ma révérence ${ }^{\mathrm{rog}}$ ", disait l'ultime phrase de la dernière lettre à Brown. Cette fragilité «déterritorialisée», au sens deleuzien, déplacée dans cet ailleurs empli d'une présence inconditionnelle - cela pourrait être du reste une juste définition de la poésie et de ses pouvoirs -, se transmue en force.

Au cœur de cette sorte de question-réponse, ce dialogue amoureux de part et d'autre du vide, ces retrouvailles au-delà de toute fin, la poésie, malgré la fragilité de l'être, apparaît quand le dernier mot même semble avoir été dit, le mot "mort" : enjambant tout ce noir, cette nuit de l'absence, elle reste ce qui, à l'instar de l'amour, est aussi fort que la mort, comme le proclamait le Cantique des cantiques. «Il est on ne peut plus facile de comprendre qu’à la fin tout devienne poésie. Le monde ne finit-il pas par devenir âme ${ }^{\text {II }}$ ?", constatait

I08. J. Keats, Lettres à Fanny et autres correspondances, ouvr. cité, p. 607.

I09. Ibid., p. 608.

IIO. Novalis, Fragments / Fragmente, ouvr. cité, p. 219. 
Novalis. Ce qui est ainsi cristallisé à la fin du film en constitue le fondement même, et toute la mélodie : comme si cette vie de Keats sous le regard de Campion était un cantique d'amour, dédié à la mystique et à la poétique de la passion, accordé à l'âme de celui qui toujours évoquait le caractère sacré de ses sentiments pour Fanny, lui écrivant en octobre I8I9 : «je pourrais devenir un martyr pour ma Religion - l'Amour est ma religion - Je pourrais mourir pour cela - je pourrais mourir pour vous. Mon Credo est l'Amour et vous en êtes l'unique article ${ }^{\text {III }}$. Une transcendance qui est exaltation de toutes les beautés, celle de l'être aimé et celle de l'univers, incarnée dans la vision aux accents panthéistes d'une déesse-étoile illuminant Bright Star de son intemporel scintillement :

Je vais imaginer que vous êtes ce soir Vénus et prier, prier, prier votre étoile comme un Païen.

À jamais vôtre, belle étoile, John Keats ${ }^{\mathrm{II}}$.

III. J. Keats, Lettres à Fanny et autres correspondances, ouvr. cité, p. 5IO. À rapprocher des sentiments de Novalis pour sa fiancée morte très jeune : "J'ai pour Sophie de la religion - non de l'amour. Un amour absolu, indépendant du cour, fondé sur la foi, est religion.» (Novalis, Fragments/Fragmente, ouvr. cité, p. 47 ; je modifie la traduction.)

II2. J. Keats, Lettres à Fanny et autres correspondances, ouvr. cité, p. 422. 